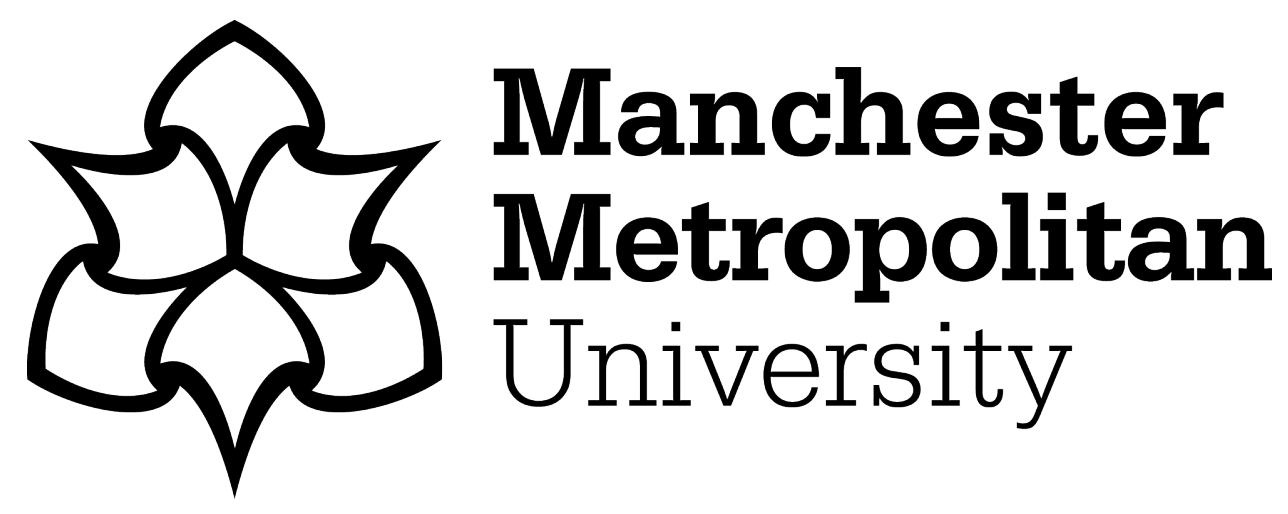

Lin, $\quad \mathrm{Xi}, \quad \mathrm{Wu}, \quad J u n$, Bashir, Ali Kashif ORCID logoORCID: https://orcid.org/0000-0001-7595-2522, Li, Jianhua, Yang, Wu and Piran, Jalil (2022) Blockchain-Based Incentive Energy-Knowledge Trading in IOT: Joint Power Transfer and AI Design. IEEE Internet of Things Journal, 9 (16). pp. 14685-14698.

Downloaded from: https://e-space.mmu.ac.uk/626669/

Version: Accepted Version

Publisher: Institute of Electrical and Electronics Engineers (IEEE)

DOI: https://doi.org/10.1109/jiot.2020.3024246

Please cite the published version 


\title{
Blockchain-Based Incentive Energy-Knowledge Trading in IoT: Joint Power Transfer and AI Design
}

\author{
Xi Lin, Jun Wu, Ali Kashif Bashir, Jianhua Li, Wu Yang, and Md. Jalil Piran
}

\begin{abstract}
Recently, edge artificial intelligence techniques (e.g., federated edge learning) are emerged to unleash the potential of big data from Internet of Things (IoT). By learning knowledge on local devices, data privacy-preserving and quality of service (QoS) are guaranteed. Nevertheless, the dilemma between the limited on-device battery capacities and the high energy demands in learning is not resolved. When the on-device battery is exhausted, the edge learning process will have to be interrupted. In this paper, we propose a novel Wirelessly Powered Edge intelliGence (WPEG) framework, which aims to achieve a stable, robust, and sustainable edge intelligence by energy harvesting (EH) methods. Firstly, we build a permissioned edge blockchain to secure the peer-to-peer (P2P) energy and knowledge sharing in our framework. To maximize edge intelligence efficiency, we then investigate the wirelessly-powered multi-agent edge learning model and design the optimal edge learning strategy. Moreover, by constructing a two-stage Stackelberg game, the underlying energy-knowledge trading incentive mechanisms are also proposed with the optimal economic incentives and power transmission strategies. Finally, simulation results show that our incentive strategies could optimize the utilities of both parties compared with classic schemes, and our optimal learning design could realize the optimal learning efficiency.
\end{abstract}

Index Terms-Edge intelligence, wireless power transfer, permissioned blockchain, incentive mechanism, game theory.

\section{INTRODUCTION}

The emerging artificial intelligence (AI) technology (e.g., machine learning) is a promising way to cope with the data explosion of IoT applications. The knowledge that AI learns from IoT data could bring many benefits to the QoS of IoT users [1]. However, the traditional cloud-centric learning paradigm faces many challenges, such as high maintenance costs, data privacy risk, and high service delay. According to [2], over 6 billion smartphones and 50 billion IoT devices will appear by 2020 with abundant under-utilized computing, communication, and storage resources. These large-scale smart devices could address cloud-centric learning challenges. Thus, on-device federated edge learning (FEL) paradigm was proposed by Google in 2016 [3]. It employs smart edge devices to perform model training in a distributed and collaborative

X. Lin, J. Wu, J. Li are with the School of Cyber Security, Shanghai Jiao Tong University, Shanghai 200240, China, and also with Shanghai Key Laboratory of Integrated Administration Technologies for Information Security, Shanghai 200240, China (e-mail: linxi234@sjtu.edu.cn; junwuhn@sjtu.edu.cn; lijh888@sjtu.edu.cn). (Corresponding author: Jun Wu).

A. K. Bashir is with the School of Computing, Mathematics, and Digital Technology, Manchester Metropolitan University, Manchester M15 6BH, U.K. (e-mail: dr.alikashif.b@ieee.org).

W. Yang is with Information Security Research Center, Harbin Engineering University, Harbin 165001, China (e-mail: yangwu@hrbeu. edu.cn).

M. J. Piran is with the department of computer science and engineering, sejong university, seoul, 05006, south korea (e-mail:piran@ sejong.ac.kr). manner, also known as "edge intelligence" or "edge AI" [4]. Only local sub-models (knowledge) rather than raw data will be uploaded and aggregated in FEL, which ensures local data privacy-preserving and better resource utilization [5] [6].

On-device FEL has many advantages over cloud-centric learning, but the learning performance is likely compromised by insufficient battery energy in traditional battery-powered edge devices, such as mobile phones, UAV (Unmanned Aerial Vehicle), and IoT devices. On the one hand, the local model training will consume limited battery power for the CPU cycles. On the other hand, edge devices are required to repeatedly communicate with the sink node (e.g., base station) to iteratively update the learning model, which also places severe demands on the battery level. Most previous works [7] [8] assume that the battery power of edge devices is sufficient to support the whole learning process, while this is not feasible in many practical scenarios. In fact, the on-device FEL process will be interrupted and smart edge devices cannot execute learning services if the battery is exhausted. So, the current edge intelligence framework is not stable and robust enough.

To prolong the battery life of smart devices, common method resorts to computation offloading to MEC (Mobile Edge Computing) nodes or other D2D (Device-to-Device) enabled devices [9] [10]. However, this method goes against the privacy-preserving feature of FEL. That is, this method could not apply to on-device FEL due to the privacy risks in learning task offloading. Another straightforward method is to employ larger batteries or recharging the batteries frequently. On one hand, increasing the battery capacity of each smart device is unrealistic, which will raise too much hardware cost for smart device manufacturers and owners. On the other hand, recharging device batteries frequently shows one of the most unfavorable features of smart devices and it is also impossible in certain situations, such as surveillance intelligent IoT nodes, where the devices are usually difficult to reach. Besides, to achieve green communication and computing in $5 \mathrm{G}$ and beyond networks, novel energy supply models need further research. The emerging energy harvesting $(\mathrm{EH})$ techniques have the potential to address the above issues well. The renewable energy from the surrounding environment could be captured, harvested, and stored (e.g., solar, wind, thermal, and electromagnetic). Benefit from EH techniques, energy-saving and sustainable computing and communication applications could be realized. Thus, by the marriage of EH techniques and edge intelligence, satisfied and sustained on-device learning performance is guaranteed.

Wireless power transfer (WPT) is one of the most promising $\mathrm{EH}$ techniques, which employs radio frequency (RF) to offer 
controllable energy supply for smart devices [11]. Compared with other techniques, WPT has higher availability, stability, and controllability. For wirelessly-powered smart devices, the received RF signal could be easily converted into stable direct current to power the batteries of smart devices. In this paper, we propose a novel Wirelessly Powered Edge intelliGence framework (WPEG), which integrates WPT to battery-constrained on-device learning. WPT brings new possibilities to edge intelligence, while still introduces some new challenges. 1) Distributed wireless power transfer is hard to guarantee security due to some selfish and untrusted behaviors (e.g., free-rider or cheating). 2) WPT improves the energy efficiency of smart devices, while consumes higher energy in the absence of an optimal charging policy. 3) WPT nodes could not actively provide charging services without some suitable monetization incentives. To resolve the above challenges, in this paper, we also employ a pemissioned blockchain approach to establish a secure P2P (Peer-to-Peer) energy trading for edge intelligence. Moreover, a two-stage Stackelberg game model is proposed to obtain the optimal transmission power strategies and incentive strategies. The contributions of our work are summarized as follows:

- We propose a novel wirelessly-powered edge intelligence framework (WPEG) to ensure the learning performance of edge intelligence in a secure and energy-saving manner.

- We establish a pemissioned edge blockchain with a lightweight consensus protocol BFT-DPoS to secure the P2P energy and knowledge sharing.

- We build the wirelessly-powered multi-agent edge learning model, including the energy-aware computing and communication model.

- We design a Stackelberg game model to simulate the energy-knowledge trading process and derive the optimal power strategies and economic incentive strategies.

The remainder of this paper is structured as follows. Section II gives an overview of the related works. Section III describes our WPEG framework, including the framework implementation and edge permissioned blockchain. In Section IV, we present the system model of our WPEG framework. Section $\mathrm{V}$ formulate and analyse the proposed Stackelberg game for energy-knowledge trading. In Section V, we conduct simulations to show the advanced performance of our framework. Finally, Section VII concludes this paper.

\section{RELATED WORK}

\section{A. Wireless Power Transfer in IoT}

Wireless power transfer (WPT) techniques employ radio frequency (RF) signals as a promising power supplying for energy-constrained IoT devices. Some researches focus on the feasibility of applying the WPT solutions to conventional communication systems. In [12], Lee et al. proposed a new WIPE protocol, which realizes sustainable communications between two devices by wireless power splitting. And a joint data-energy beamforming and SWIPT-based traffic offloading scheme [13] has been proposed for C-RANs via D2D communications. Besides, a channel coding-based IoT communication network with WPT stabilization algorithms has been presented in [14]. Some other works introduced WPT technologies into mobile edge computing (MEC) systems. In [15], the authors studied a multi-user MEC system with WPT functions, and jointly optimized the computation offloading and transmission time allocation in the system. And an energy-effective cooperative resource allocation policy for the WPT-MEC system was also proposed in [16] to guarantee the fairness of mobile users. In addition, Liu et al. in [17] presented a UAV-assisted WPT-MEC system, and studied the joint optimization of task offloading, CPU control, and trajectory design. There are still some existing works considering cache-aided WPT-IoT networks. In [18], Gautam et al. investigated the relay selection strategy in a cache-aided SWIPT framework with a dynamic time switching (TS). And a sleeping mechanism for cacheaided heterogeneous networks with energy harvesting methods has been presented in [19].

In general, wirelessly-powered IoT networks have been well studied, but are limited to wirelessly-powered communication, computing, and caching in IoT networks. Although the combination of WPT technologies with the emerging edge AI technologies could improve the edge learning performance in IoT, which is ignored by the previous works.

\section{B. Edge Intelligence for IoT}

Combined with edge computing, the emerging edge AI has already become the last mile of artificial intelligence for IoT [20] [21]. Once appeared, it received a tremendous amount of interest. In terms of edge AI algorithms, in [22] [23], Li et al. proposed an Edgent framework, which leverages deviceedge synergy computing to accelerate training and inference of deep neural network (DNN). And the authors in [24] design a momentum federated learning (MFL) framework to accelerate federated learning by momentum gradient descent. In addition, a sparse ternary compression (STC) framework [25] was designed for federated learning with non-i.i.d data. Moreover, in [26], Zhou et al. employed the Lyapunov optimization theory to design an online cost-efficient federated learning optimization framework named CEFL under the dynamic training data arrive. For resource sharing in edge AI, an efficient and incentive knowledge market was proposed in [27], which achieves secure and efficient edge knowledge sharing and exchange for edge AI. In [28] [29], the authors proposed a privacy-preserved data sharing scheme in industrial IoT (IIoT). In [30], a secure and robust federated learning scheme with differential privacy has been proposed for urban informatics.

Some existing works [7] [8] assumed the energy of edge devices could support the computing-intensive learning task, which is not realistic. Others [31] [32] adopted the energy as a constraint condition for task execution, which results in limitations in learning performance. Compared with existing works, our WPEG framework not only could achieve a stable, robust, and sustainable edge learning, but also improve the efficiency and performance of edge AI.

\section{Blockchain for IoT}

Blockchain is a feasible tool to establish distributed trust among IoT devices. In [33], Yang et al. proposed a blockchain- 


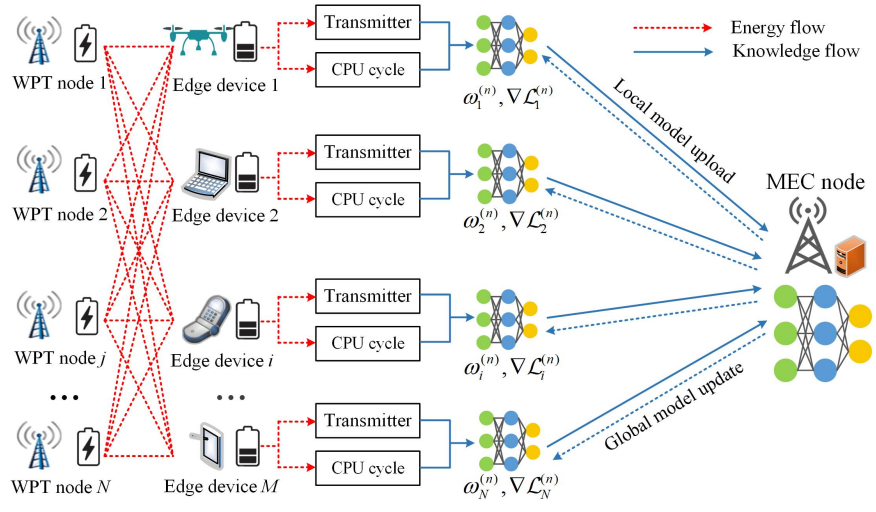

Fig. 1: The framework of our proposed WPEG.

based decentralized trust management system in vehicular networks. And a blockchain-based trustworthy computing resource trading system in vehicular networks has been discussed in [34]. For IIoT, Li et al. [35] built a consortium energy blockchain with a credit-based payment scheme to realize fast and frequent energy trading. And Zhang et al. [29] designed an edge-AI and blockchain enabled IIoT framework, which supports flexible and secure edge service management. In terms of data security, Xu et al. [36] proposed a novel blockchain-based trustworthy edge caching scheme for cyberphysical systems (CPSs). And the authors in [28] incorporated differential privacy into permissioned blockchain for privacy-preserved data sharing in IIoT. Some other works focus on blockchain-based incentive mechanisms, a consortium blockchain-based efficient and incentive Approach for IoT knowledge sharing has been presented in [27]. And the authors in [37] investigated the incentive mechanism for rational miners in edge-computing-enable blockchain systems. Moreover, a Stackelberg game-based incentive computing resource management has been studied in [38] for proof-of-work (PoW) based public blockchain networks.

Some previous works mainly focused on classic blockchain consensus protocols (such as PoW [37] [38]), while lightweight consensus protocols for edge blockchain systems (such as DPoS) have not been well applied. Besides, previous works only considered a single type of resource sharing (such as knowledge [27] or energy [35]), while multi-resource sharing did not involve.

\section{WIRELESSLY-POWERED EdGE INTELLIGENCE FRAMEWORK}

We propose the novel WPEG framework in this section. First, we introduce the main elements of the WPEG framework and show how they interact in detail. Then, we provide the details of the proposed permissioned edge blockchain in our WPEG framework.

\section{A. The Elements in Our WPEG Framework}

We consider our WPEG framework is deployed at the edge of networks, which includes geo-distributed MEC (Mobile Edge Computing) nodes, large-scale edge devices and WPT
(Wireless Power Transfer) nodes, as shown in Fig. 1. Each upstream MEC node is responsible for managing edge devices and WPT nodes within its coverage. The WPEG framework mainly contains the following elements.

- WPT nodes: We deploy a certain number of wireless charging stations as infrastructure in the edge network environment. These WPT nodes could not only obtain energy from the traditional power grid, but also have the ability to harvest renewable energy from the surrounding environment (e.g., solar and wind) with $\mathrm{EH}$ equipment. The energy stored in WPT nodes will charge edge devices via wireless channels. We consider two possible transmission paths: 1) Stationary mode: when edge devices are close to the static power beacon, then it will start to emit radio waves to supplement energy; 2) Dynamic mode: the power beacon is deployed on mobile objects (e.g., drones and vehicles) and charges edge devices in a dynamic manner. Our WPEG framework implementation could employ one or two of the above models for supplement wireless energy to edge devices.

- Edge devices: Edge devices, such as smart phones, UAVs, IoT intelligent sensors, and microservers (Raspberry Pi), are required to provide edge AI services (i.e., federated edge learning) through local data training. Edge devices are energy-constrained so that they are difficult to guarantee a stable and robust learning performance. Thus they will obtain the wireless energy from WPT nodes. On the one hand, they utilize the wireless energy for executing model training (CPU cycle); On the other hand, they employ the wireless energy to repeatedly communicate (transmitter) with the upper MEC node for global learning model updating.

- MEC nodes: The MEC node first serves as the mediation between the edge AI service requester and edge devices. That is, the service requester will publish tasks, design optimal learning strategy, and reward the contribution of edge learning through MEC nodes. Besides, the local MEC node plays the role of aggregator in federated edge learning, which is responsible for collecting, aggregating, and updating the trained local sub-models from edge devices. Moreover, in order to ensure the security features of the entire framework, an edge permissioned blockchain will be established between MEC nodes to guarantee the secure $\mathrm{P} 2 \mathrm{P}$ wireless energy sharing and knowledge (model) sharing.

In Fig. 2, we show that how the elements interact with each other, and also present how our WPEG framework works. As shown in Fig. 2, the interactive process is divided into 5 steps:

- Step 1: Edge AI service requesters will publish edge learning tasks through MEC nodes, and set corresponding learning parameters (e.g., local/global training accuracy) and service time limits. Then the MEC node will select the corresponding edge devices to perform the learning task according to the service type and resource status of the local edge devices. 


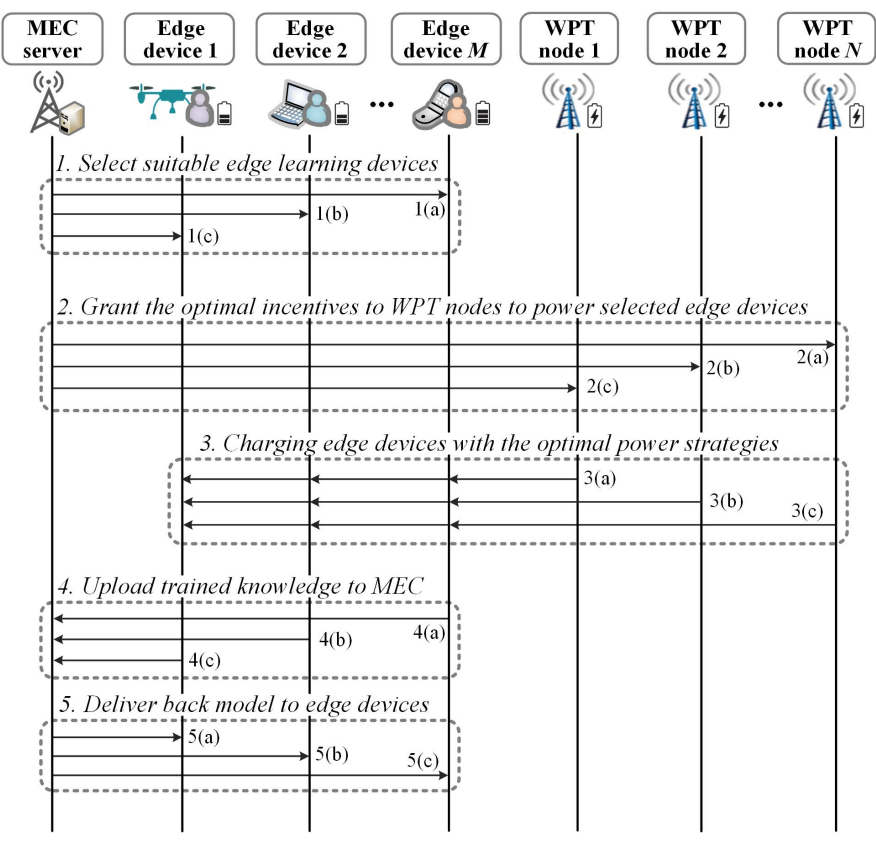

Fig. 2: The interaction of elements in our WPEG framework.

- Step 2: Due to the limited energy of edge devices, it is difficult to complete the whole learning task. In order to obtain training model (knowledge) from edge devices, the edge AI service requester needs to incentivize local WPT nodes to power edge devices, so that edge device can ensure the stable and robust learning process. We believe that WPT nodes are selfish and will not provide charging services for free. Thus this process requires edge AI service requesters to provide appropriate economic incentives for WPT owners through the MEC node. The details about economic incentives are given in Section V.

- Step 3: WPT nodes will charge edge devices under the economic incentives. In order to avoid wasting energy during this process, it is necessary to obtain the optimal power transmission strategy according to energy demands in the edge learning process. At the same time, it also needs to solve the security problems in $\mathrm{P} 2 \mathrm{P}$ wireless energy sharing (e.g., free riding). The details about optimal power transmission strategy are given in Section V.

- Step 4 and Step 5: Under the predetermined time limit and learning accuracy requirements, edge devices will adopt the received energy from WPT nodes to implement federal edge learning, and upload the trained sub-models (knowledge) to the MEC server for aggregation. Then the MEC node will update the global model and deliver it back to all the edge devices. Step 4 and Step 5 are performed in a periodic manner. Eventually, the MEC node feeds the trained global model back to the edge AI service requester.

It should be noted that the service requesters also need to give corresponding rewards to edge devices, which can be considered as knowledge service trading. The corresponding reward mainly depends on the data contribution of edge devices in the learning process, which has been discussed in our previous works [27]. In the following text, we believe that edge devices have already decided the optimal data contribution $d_{i}^{*}$, and the service requester has designed the optimal knowledge service pricing strategy.

\section{B. Permissioned Blockchain in Our WPEG Framework}

As shown in Fig. 3, there are two trading modes in our framework, i.e., wireless energy trading and knowledge service trading. And the process of both energy and knowledge sharing will carry out in a Peer-to-Peer (P2P) manner. We believe that all parties in the trading process are untrusted individuals. Thus our framework will face many security attacks. For example, the free-rider, greedy and cheating energy (or knowledge) sharing phenomenon will happen in our framework. That is, on the one hand, some untrusted edge devices will deny the reception of wireless energy, which will cause great energy consumption of WPT nodes; on the other hand, the service requester may deny the acquired knowledge services, which damages the profit of edge devices. Besides, the traditional centralized trading management system also faces the single point of failure, distributed denial of service (DDoS) and Sybil attacks, which will greatly affect the security and system efficiency of our framework.

The emerging blockchain technology has become a promising distributed solution for implementing secure P2P trading. It does not rely on any centralized entity and has gained wide attention from academia and industry for its anonymity, security, and traceability. Motivated by this, we adopt blockchain technologies to defend against the security threats in our framework. Specifically, we build an edge permissioned (consortium) blockchain as a distributed energy/knowledge trading management platform, which could also encourage both parties to participate in our framework under a security guarantee. Unlike the previous work [35] [39], we employ a more lightweight and efficient consensus protocol BFTDPoS (Byzantine Fault Tolerance-Delegated Proof of Stake) in resource-constrained edge environment, which is popular in EOS project. The proposed edge permissioned blockchain based on the BFT-DPoS consensus protocol works as the following phases:

Phase 1. Market initialization: Each entity (e.g., edge devices, WPT nodes, and MEC nodes) will first be authorized by TA (Trust Authority) to becomes a legitimate participant in the trading market. That is, TA will verify the service capabilities of each entity, and only entities that meet the service requirements can join our market. Besides, each authorized entity $i$ will obtain corresponding asymmetric keys $\left\{P K_{i}^{k}, S K_{i}^{k}\right\}$ and certificates $\left\{\operatorname{Cert}_{i}^{k}\right\}$ from TA. Moreover, each participant will have a wallet account $\left\{\right.$ Wallet $\left._{i}^{k}\right\}$ to manage their cryptocurrency.

Phase 2. Miner group formation: The local MEC node is responsible for recording and managing local transaction data as shown in Fig. 3. And MEC nodes also act as miners of the blockchain to complete the consensus process. The MEC node applies for being a miner by submitting a deposit (stakes) to the blockchain system. If the MEC node performs malicious operations in the subsequent consensus process, this deposit will be cleared. 


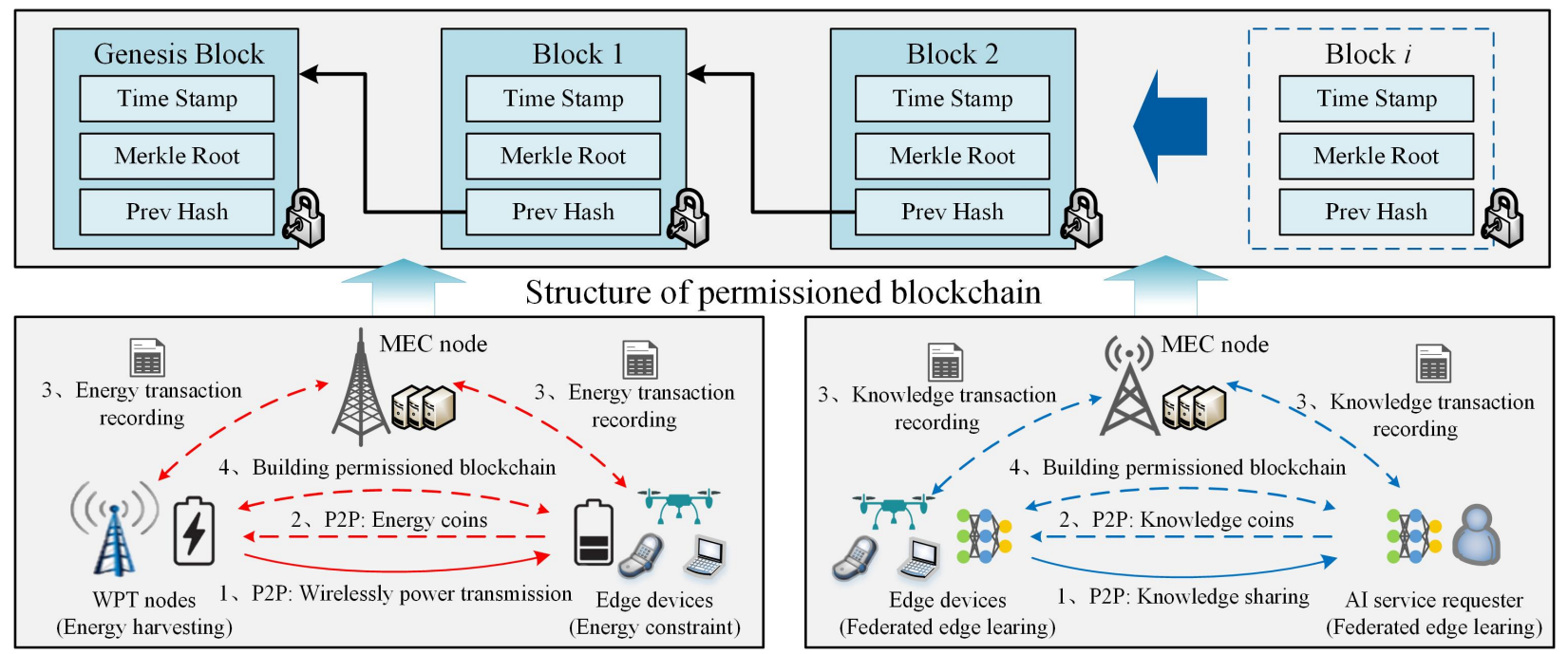

Fig. 3: The permissioned blockchain in our proposed WPEG.

Phase 3. Delegated miner selection: Each other entity (i.e., WPT nodes and edge devices) has its own stakes, thus they could vote for the miners and select their delegates. The top-k miners of the miner group with high stakes could be selected as the delegates of our blockchain, where $k$ is an odd integer (e.g., $k=21$ in EOS project). These delegates are responsible for the generation, management and mutual verification of transaction blocks in our blockchain. And the selection of delegates will be conducted in a periodic manner.

Phase 4. BFT consensus process: In order to clearly illustrate the consensus process, we still take the EOS project as an example. In the traditional DPoS consensus process, a delegate is randomly selected as the leader to generate a transaction block. We assume the block generation time is 3 seconds in $E O S$ project, thus it will take 45 seconds to confirm the transaction in this block. Because the transaction confirmation requires more than $2 / 3$ delegates (i.e., 14 delegates) to verify. Only when other delegates generate new blocks, the confirmation of the entire previous blockchain is completed. So it will take 45 seconds to confirm the transaction in the DPoS protocol. To further improve performance, we introduced the BFT mechanism in the consensus process. The selected leader still broadcasts the new block, but other delegates immediately verify the block after receiving this block, and return the results with the verified signature to the leader. From the perspective of the current leader, it produces a new block and broadcast it to the whole network, and then received confirmation from other delegates. If $2 / 3$ delegates have the consistent results. The transaction in this block is irreversible. The transaction confirmation time is greatly shortened, from 45 seconds to about 3 seconds. After all the delegates complete the block consensus process, the blockchain system will give corresponding cryptocurrency rewards to their wallet.

The energy/knowledge trading is shown in Fig. 3, which is presented as follows: energy/knowledge traders first obtain $\left\{P K_{i}^{k}, S K_{i}^{k}\right\},\left\{\operatorname{Cert}_{i}^{k}\right\}$, and $\left\{W\right.$ allet $\left.t_{i}^{k}\right\}$ via market initialization. Authorized traders with legal certificates can access the local MEC transaction server to initiate a transaction.
The local MEC server could match the supply and demand side of the resource. After wirelessly power transfer (or knowledge sharing), the energy/knowledge buyer needs to pay for corresponding rewards (i.e., energy coins or knowledge coins). Specifically, a new transaction will be generated by traders and recorded by the local MEC server for audit. All the MEC servers will establish a permissioned edge blockchain for cross-auditing and mutual verification, which adopts the lightweight BFT-DPoS protocol for cross-domain consensus.

\section{SySTEM MODEL}

In this section, we model the wirelessly-powered multiagent learning process and formulate the utility function of both parties. In our proposed WPEG framework, we consider there exists $M$ edge devices and $N$ WPT nodes, which are represented as $\mathcal{M}=\{1,2, \ldots, i, \ldots, M\}$ and $\mathcal{N}=$ $\{1,2, \ldots, j, \ldots, N\}$. All the edge devices are equipped with $\mathrm{EH}$ components and completely powered by the collected wireless energy from WPT nodes. Federated learning mainly contains two parts: local training and global updating. For each local training, it can be subdivided into the computing (training) process and the communication (uploading) process.

\section{A. Preliminaries of Federated Learning}

In federated learning, each edge device $i(\forall i \in \mathcal{M})$ owns a local personal dataset $\mathcal{D}_{i}$. And the size of data samples in $\mathcal{D}_{i}$ is denoted as $d_{i}$. Each data sample contain$\mathrm{S}$ an input-output pair $\left\{\boldsymbol{x}_{k}, \boldsymbol{y}_{k}\right\}_{k=1}^{d_{i}}$, where the input $\boldsymbol{x}_{k}$ is a sample vector with various features, and the output $\boldsymbol{y}_{k}$ is the label value of the input features from users' mobile application. The loss function of federated learning is defined as $f_{k}\left(\boldsymbol{x}_{k}, \boldsymbol{y}_{k}, \boldsymbol{\omega}\right)$. Some example loss functions are $f_{k}\left(\boldsymbol{x}_{k}, \boldsymbol{y}_{k}, \boldsymbol{\omega}\right)=0.5\left\|\boldsymbol{y}_{k}-\boldsymbol{\omega}^{T} \boldsymbol{x}_{k}\right\|^{2}$ for linear regression and $f_{k}\left(\boldsymbol{x}_{k}, \boldsymbol{y}_{k}, \boldsymbol{\omega}\right)=0.5 \lambda\|\boldsymbol{\omega}\|^{2}+0.5 \max \left\{0 ; 1-\boldsymbol{y}_{k} \boldsymbol{\omega}^{T} \boldsymbol{x}_{k}\right\}^{2}$ for squared-SVM. For each edge device with dataset $\mathcal{D}_{i}$, the local average loss function is

$$
\mathcal{L}_{i}(\boldsymbol{\omega})=\frac{1}{\left|\mathcal{D}_{i}\right|} \sum_{k=1}^{k=d_{i}} f_{k}\left(\boldsymbol{x}_{k}, \boldsymbol{y}_{k}, \boldsymbol{\omega}\right)
$$


Thus, the loss function minimization problem of global learning process could be described as follows:

$$
\min _{\boldsymbol{\omega}^{*}} \mathcal{L}_{\text {gobal }}(\boldsymbol{\omega})=\frac{\left|\mathcal{D}_{i}\right| \sum_{i \in \mathcal{M}} \mathcal{L}_{i}(\boldsymbol{\omega})}{\sum_{i \in \mathcal{M}}\left|\mathcal{D}_{i}\right|}
$$

1) Local training: Each edge device $i$ solves the local loss function minimization problem with a local accuracy $\delta_{i}(0 \leq$ $\left.\delta_{i} \leq 1\right)$ as follows:

$$
\boldsymbol{\omega}_{i}^{(n)}=\arg \min \mathcal{L}_{i}\left(\boldsymbol{\omega}_{i} \mid \boldsymbol{\omega}_{i}^{(n-1)}, \nabla \mathcal{L}^{(n-1)}\left(\boldsymbol{\omega}_{i}\right)\right)
$$

Where $\left\|\nabla \mathcal{L}_{i}\left(\boldsymbol{\omega}_{i}^{(n)}\right)\right\| \leq \delta_{i}\left\|\nabla \mathcal{L}_{i}\left(\boldsymbol{\omega}_{i}^{(n-1)}\right)\right\|$ at the $n^{\text {th }}$ model update. When $\delta_{i}=0$, it shows the local problem has been solved optimally, while $\delta_{i}=1$ means no progress at all in the local training [5] [40]. Each edge device then uploads weights $\boldsymbol{\omega}_{i}^{(n)}$ and gradients $\nabla \mathcal{L}_{i}{ }^{(n)}$ to the MEC node for aggregating the global model.

2) Global updating: The MEC node collects and aggregates the uploading sub-models from edge devices as:

$$
\boldsymbol{\omega}^{(n+1)}=\frac{1}{M} \sum_{i \in \mathcal{M}} \boldsymbol{\omega}_{i}^{(n)}, \nabla \mathcal{L}^{(n+1)}=\frac{1}{M} \sum_{i \in \mathcal{M}} \nabla \mathcal{L}_{i}^{(n)}
$$

Then the MEC node will send the global model back to all the edge devices. The interactive learning process will be carried out in an iterative manner. Until $\left\|\nabla \mathcal{L}\left(\boldsymbol{\omega}^{(n)}\right)\right\| \leq$ $\alpha\left\|\nabla \mathcal{L}\left(\boldsymbol{\omega}^{(n-1)}\right)\right\|$ is realized, the global iteration will stop. Where $\alpha \in[0,1]$ represents the global model accuracy. Due to the convex objective $\mathcal{L}_{i}$ and $\mathcal{L}_{\text {global }}$, the local iterations is general upper bounded to $\mathcal{O}\left(\log \left(1 / \delta_{i}\right)\right)$, which is applicable for a wide range of iterative algorithms (e.g., gradient or stochastic descent) [5] [40]. Besides, the global iterations could be calculated as $\mathcal{O}(\log (1 / \alpha)) /\left(1-\delta_{i}\right)$, which is very intuitive. When global accuracy $\alpha$ is bigger (lower accuracy), the federated learning systems need to runs less global iterations. And when local accuracy $\delta_{i}$ is bigger (lower accuracy), the federated learning systems need to runs more global iterations.

\section{B. Wirelessly-Powered Computing Model for Learning}

In our WPEG, we assume each WPT node $j(j \in \mathcal{N})$ will provide controllable and constant RF energy transfers for each edge device $i(i \in \mathcal{M})$. And the transmission power is shown as $\boldsymbol{\theta}=\left(\theta_{1}, \theta_{2}, \ldots, \theta_{j}, \ldots, \theta_{N}\right)$. We believe that the MEC node could schedule spectrum resources well, i.e., the RF energy transmission and sub-models uploading will work in different frequency bands, thus the signal interference by edge devices could be ignored. The amount of energy harvested by edge devices only depends on the transmission power $\boldsymbol{\theta}$ and the channel power gain $h_{j i}(\forall j \in \mathcal{N}, i \in \mathcal{M})$. We employ a typical linear energy harvesting model like previous works [41]. Therefore, the harvested energy by edge device $i$ from WPT nodes could be shown as:

$$
E=\kappa_{i} T \sum_{j \in N} h_{j i} \theta_{j}=\Theta_{i} T, \forall i \in \mathcal{M}
$$

Where $E$ and $T$ represents energy consumption and transmission time, respectively. And $\kappa_{i} \in[0,1]$ shows the energy harvesting efficiency.
We assume each edge device $i$ will contribute the CPU computing resource $f_{i} \in\left[0, f_{i}^{\max }\right]$ in the local training process. And the number of CPU cycles for edge device $i$ to train one sample of local data is $c_{i}$. Thus, the local computing time $T_{i}^{c m p}$ is equal to $\mathcal{O}\left(\log \left(1 / \delta_{i}\right)\right) c_{i} d_{i} / f_{i}$. And the energy consumption of local training $E_{i}^{c m p}$ is equal to $\mathcal{O}\left(\log \left(1 / \delta_{i}\right)\right) \mu_{i} c_{i} d_{i} f_{i}^{2}$, where the $\mu_{i}$ is the effective capacitance coefficient of edge device $i$. We consider that all the computing energy will be supplied by the RF energy from WPT nodes, thus we can get:

$$
E_{i}^{c m p}\left(f_{i}\right)=\Theta_{i} T_{i}^{c m p}\left(f_{i}\right), \forall i \in \mathcal{M}
$$

By substitute $T_{i}^{c m p}$ and $E_{i}^{c m p}$ into Eq. (6), we could obtain the computing resource scheduling strategy $f_{i}$ as:

$$
f_{i}\left(\Theta_{i}\right)=\min \left[\sqrt[3]{\frac{\kappa_{i}}{\mu_{i}} \sum_{j \in \mathcal{N}} h_{j i} \theta_{j}}, f_{i}^{\max }\right], \forall i \in \mathcal{M}
$$

That is, in the EH-enable training process, edge devices need to adjust own computing resources to meet the energy constraints. It can be achieved by changing the voltage of computing chipset with DVFS (Dynamic Voltage and Frequency Scaling) techniques. Moreover, we could derive the $T_{i}^{c m p}$ and $E_{i}^{c m p}$ in local training process as follow:

$$
\begin{aligned}
T_{i}^{c m p}\left(\Theta_{i}\right) & =\mathcal{O}\left(\log \left(\frac{1}{\delta_{i}}\right)\right) c_{i} d_{i} \sqrt[3]{\frac{\mu_{i}}{\kappa_{i} \sum_{j \in \mathcal{N}} h_{j i} \theta_{j}}} \\
E_{i}^{c m p}\left(\Theta_{i}\right) & =\mathcal{O}\left(\log \left(\frac{1}{\delta_{i}}\right)\right) c_{i} d_{i} \sqrt[3]{\mu_{i} \kappa_{i}^{2}\left(\sum_{j \in \mathcal{N}} h_{j i} \theta_{j}\right)^{2}}
\end{aligned}
$$

\section{Wirelessly-Powered Communication Model for Learning}

After completing the local training, each edge device then uploads sub-models to the MEC node via the wireless uplink. Similarly, the required energy for communication is completely provided by the RF energy. Based on Eq. (5), the uplink transmit power of edge device $i$ is $P_{i}=E_{i}^{c o m} / T_{i}^{c o m}=\Theta_{i}$. Thus the data rate (bit/s) of edge device $i$ is shown as:

$$
r_{i}=B \log _{2}\left(1+\frac{P_{i} H_{i}}{N_{0} B}\right)=B \log _{2}\left(1+\frac{\kappa_{i} H_{i}}{N_{0} B} \sum_{j \in \mathcal{N}} h_{j i} \theta_{j}\right)
$$

Where $B$ represents the bandwidth, $H_{i}$ shows the wireless channel gain between edge device $i$ and the MEC node, and $N_{0}$ denotes the noise power spectrum density.

We assume the data size of both local weights $\boldsymbol{\omega}_{i}^{(n)}$ and local gradients $\nabla \mathcal{L}_{i}{ }^{(n)}$ is $\rho_{i}$. Thus the communication time $T_{i}^{c o m}$ and communication energy consumption $E_{i}^{c o m}$ could be described as follows:

$$
\begin{gathered}
T_{i}^{c o m}=\rho_{i} / r_{i}=\frac{\rho_{i}}{B \log _{2}\left(1+\frac{\kappa_{i} H_{i}}{N_{0} B} \sum_{j \in \mathcal{N}} h_{j i} \theta_{j}\right)} \\
E_{i}^{c o m}=P_{i} T_{i}^{c o m}=\frac{\rho_{i} \kappa_{i} \sum_{j \in \mathcal{N}} h_{j i} \theta_{j}}{B \log _{2}\left(1+\frac{\kappa_{i} H_{i}}{N_{0} B} \sum_{j \in \mathcal{N}} h_{j i} \theta_{j}\right)}
\end{gathered}
$$

It should be noted that when the global model is fed back from the MEC node to edge devices, we ignore the downlink communication time and energy consumption. This is because the downlink rate from the MEC node to edge devices is higher 
TABLE I: Main Terms Referred in Our Framework

\begin{tabular}{ll}
\hline Symbol & Explanations \\
\hline $\mathcal{M}$ & Edge device set to execute learning tasks \\
$\mathcal{N}$ & WPT node set to charge edge devices \\
$c_{i}$ & The CPU cycles number required for one \\
& sample data training for edge device $i$ \\
$d_{i}$ & The data size of local personal dataset of \\
& edge device $i$ \\
$\delta_{i}$ & The local sub-model trained accuracy of \\
& edge device $i$
\end{tabular}

than uplink rate from edge devices to BS. Meanwhile, the MEC node is not subject to energy restrictions.

\section{Utility Function Formulation}

In our WPEG, we consider that WPT nodes are conditional volunteers. That is, power transmission can only be carried out if the corresponding economic incentives are obtained. We assume service requesters will publish federated learning tasks through the MEC node, and give WPT nodes economic rewards. The rewards for unit power transmission of WPT nodes are defined as $\boldsymbol{R}=\left(R_{1}, R_{2}, \ldots, R_{j}, \ldots, R_{N}\right)$. Thus, we could formulate the utility function of WPT nodes and the MEC node (service requesters). The main symbols and explanations in WPEG framework are shown in TABLE 1.

1) Utility function of WPT nodes: On the one hand, WPT nodes could obtain rewards from the MEC node, on the other hand, they still need to pay the corresponding energy costs to the energy supplier. In our paper, we employ the typical quadratic function model to represent the energy cost of WPT nodes [42]. Therefore, the utility function of WPT nodes is described as follows:

$$
U_{j}^{W P T}\left(R_{j}, \theta_{j}\right)=R_{j} \theta_{j}-\left(a_{j} \theta_{j}^{2}+b_{j} \theta_{j}+e_{j}\right), \forall j \in \mathcal{N}
$$

Where $a_{j}, b_{j}, e_{j}$ are the energy cost factors of the WPT node $j(\forall j \in \mathcal{N})$. Besides, $a_{j}, b_{j}$ are positive constants for each WPT node $j$, and $e_{j}=0$.

2) Utility function of the MEC node: In order to obtain a trained AI model (i.e., knowledge) from edge devices, the MEC node needs to give economic incentives to WPT nodes for charging. While the profit of the MEC node lies in the quality of the local AI training service. We assume that the MEC node will pre-set a deadline $T_{i}^{\max }$ for completing AI training sub-tasks for each edge device $i$. The quality of the local AI training service of edge device $i$ could be defined as:

$$
\varphi_{i}\left(\Theta_{i}\right)=T_{i}^{\max }-T_{i}^{\text {learn }}\left(\Theta_{i}\right), \forall i \in \mathcal{M}
$$

Where $T_{i}^{\text {learn }}\left(\Theta_{i}\right)=T_{i}^{c m p}\left(\Theta_{i}\right)+T_{i}^{\text {com }}\left(\Theta_{i}\right)$ represents the total time for local training. Besides, to realize the proportional fairness among edge devices, we utilize typical logarithm utility functions [34] to capture the satisfaction degree of $\varphi_{i}\left(\Theta_{i}\right)$, i.e., $S_{i}\left(\Theta_{i}\right)=\xi_{i} \ln \left(1+\varphi_{i}\left(\Theta_{i}\right)\right)$. The satisfaction degree $S_{i}\left(\Theta_{i}\right)$ of the local AI service is considered as the obtain profit of the MEC node. Therefore, the utility of the MEC node is denoted as the total profits minus the incentive costs, which is shown as:

$$
U^{M E C}(\boldsymbol{R}, \boldsymbol{\theta})=\sum_{i \in \mathcal{M}} \xi_{i} \ln \left(1+\varphi_{i}\left(\Theta_{i}\right)\right)-\sum_{j \in \mathcal{N}} R_{j} \theta_{j}
$$

\section{Stackelberg Game Formulation and Analysis}

In our WPEG, we need to obtain the optimal power transmission and economic rewards to joint maximize the utility of both WPT nodes and the MEC node. To solve the joint optimization problems, we model the interaction between WPT nodes and the MEC node as a two-stage Stackelberg game. This two-stage game could be considered as energyknowledge trading. That is, to obtain knowledge derived from the local data of edge devices, the MEC node needs to purchase energy from WPT nodes. At stage I, the MEC node acts as a leader in the upper game who sets economic reward strategies for each WPT node. At stage II, WPT nodes can be considered as followers in the sub-game who determine their power transmission strategies according to the given economic rewards. In general, we formulate our Stackelberg game model $\mathbb{G}=\left\{S_{i \in \mathcal{M}}^{M E C}, S_{j \in \mathcal{N}}^{W P T} ; U_{i \in \mathcal{M}}^{M E C}, U_{j \in \mathcal{N}}^{W P T}\right\}$ as shown in the following:

- $S_{j \in \mathcal{N}}^{W P T}$ shows the strategy space of WPT nodes. The power transmission strategies of WPT nodes are $\theta=$ $\left(\theta_{1}, \theta_{2}, \ldots, \theta_{j}, \ldots, \theta_{N}\right)(\forall j \in \mathcal{N})$. Thus, we obtain that $S_{j \in N}^{W P T}=\left\{\theta_{j} \mid 0 \leq \theta_{j} \leq \theta_{j}^{\max } ; \forall j \in \mathcal{N}\right\}$. The transmission power $\theta_{j}$ should be non-negative and not to exceed the maximum transmission power $\theta_{j}^{\max }$. Similarly, $S_{i \in \mathcal{M}}^{M E C}$ denotes the incentive strategy space of the MEC node. The strategy space could be defined as $S_{i \in \mathcal{M}}^{M E C}=\left\{R_{j} \mid R_{j}^{\min } \leq R_{j} \leq R_{j}^{\max } ; \forall i \in \mathcal{M}, j \in \mathcal{N}\right\}$.

- $U_{j \in \mathcal{N}}^{W P T}\left(R_{j}, \theta_{j}\right)$ is the utility function of the followers (i.e., WPT nodes), and $U_{i \in \mathcal{M}}^{M E C}(\boldsymbol{R}, \boldsymbol{\theta})$ is the utility function of the leader (i.e., the MEC node).

According to the above, at stage I, the objective optimization problem P1 of the upper game for the MEC node could be described as follows: 


$$
\begin{aligned}
\max _{(\boldsymbol{\theta}, \boldsymbol{R})} U^{M E C}= & \sum_{i \in \mathcal{M}} \xi_{i} \ln \left[T_{i}^{\max }-\frac{\rho_{i}}{B \log _{2}\left(1+\frac{\kappa_{i} H_{i}}{N_{0} B} \sum h_{j i} \theta_{j}\right)}\right. \\
& \left.-\mathcal{O}\left(\log \left(\frac{1}{\delta_{i}}\right)\right) \sqrt[3]{\frac{\mu_{i} c_{i}^{3} d_{i}^{3}}{\kappa_{i} \sum h_{j i} \theta_{j}}}+1\right]-\sum_{j \in \mathcal{N}} R_{j} \theta_{j} \\
\text { s.t. } & R_{j}^{\min } \leq R_{j} \leq R_{j}^{\max }, \forall j \in \mathcal{N} \\
& \theta_{j}^{\min } \leq \theta_{j} \leq \theta_{j}^{\max }, \forall j \in \mathcal{N} \\
& 0<\delta_{i}<1, \forall i \in \mathcal{M}
\end{aligned}
$$

In addition, at stage II, the objective optimization problem P2 of the sub-game for WPT nodes could be formulated as:

$$
\begin{aligned}
\max _{\left(\theta_{j}, R_{j}\right)} & U_{j}^{W P T}=R_{j} \theta_{j}-\left(a_{j} \theta_{j}^{2}+b_{j} \theta_{j}+e_{j}\right) \\
\text { s.t. } & R_{j}^{\min } \leq R_{j} \leq R_{j}^{\max }, \forall j \in \mathcal{N} \\
& \theta_{j}^{\min } \leq \theta_{j} \leq \theta_{j}^{\max }, \forall j \in \mathcal{N} \\
& 0<\delta_{i}<1, \forall i \in \mathcal{M}
\end{aligned}
$$

It should be noted that the edge network status is dynamic, and some important information (e.g., channel conditions) will change with time. Thus, the Stackelberg game will perform periodic operations. After one period, the power transmission strategy and economic incentive strategy will be re-designed. We assume that WPT nodes will send channel conditions and energy cost factors to the MEC node, and edge devices will send the relevant learning parameters to the MEC node. So that the MEC node has complete information to derive the optimal strategy. And the scenario of asymmetric information is our future work.

\section{A. Sub-Game of WPT Nodes}

We apply the backward induction method to solve the joint problems (i.e., P1 and P2). So we first analyse the power transmission sub-game of WPT nodes. The sub-game could be considered as a non-cooperative game between WPT nodes, and WPT nodes could dynamically adjusts the power transmission strategies to optimize the self-revenue.

Definition 1 (NE): In sub-game, the power transmission strategies $\boldsymbol{\theta}^{*}=\left(\theta_{1}^{*}, \theta_{2}^{*}, \ldots, \theta_{j}^{*}, \ldots, \theta_{N}^{*}\right)$ of WPT nodes is the Nash equilibrium (NE), if and only if $U_{j}^{W P T}\left(R_{j}^{*}, \theta_{j}, \Theta_{-j}\right) \leq$ $U_{j}^{W P T}\left(R_{j}^{*}, \theta_{j}^{*}, \Theta_{-j}\right)$, where $\Theta_{-j}$ is the power transmission strategy set of WPT nodes excluding $\theta_{j}$.

Theorem 1: A unique Nash equilibrium exists in sub-game. And the optimal transmission power $\theta_{j}$ of WPT node $j^{*}$ is:

$$
\theta_{j}^{*}=\frac{R_{j}-b_{j}}{2 a_{j}}, \quad \forall j \in \mathcal{N}
$$

Proof: We find the first and second derivative of the utility function $U_{j}^{W P T}$ with respect to $\theta_{j}$ as the following:

$$
\begin{gathered}
\frac{\partial U_{j}^{W P T}}{\partial \theta_{j}}=R_{j}-2 a_{j} \theta_{j}-b_{j}, \forall j \in \mathcal{N} \\
\frac{\partial^{2} U_{j}^{W P T}}{\partial^{2} \theta_{j}}=-2 a_{j}<0, \forall j \in \mathcal{N}
\end{gathered}
$$

$U_{j}^{W P T}$ is a strictly concave function about $\theta_{j}$. And due to the boundedness of $\theta_{j}, \mathbf{P 2}$ should be a strict convex optimization problem. Thus, a unique Nash equilibrium exists in sub-game. By solving $\partial U_{j}^{W P T} / \partial \theta_{j}=0$, we could obtain Eq. (16).

Lemma 1: The optimal transmission power $R_{j}^{*}$ of WPT node $j$ satisfies $b_{j} \leq R_{j}^{*} \leq 2 a_{j} \theta_{j}^{\max }+b_{j}$.

Proof: According to the definition of transmission power, we could have $\theta_{j}^{*} \geq 0$. Thus, $\theta_{j}^{*}=\left(R_{j}^{*}-b_{j}\right) / 2 a_{j} \geq 0$ and $R_{j}^{*} \geq b_{j}$ based on the Eq. (16). The lower bound of $R_{j}^{*}$ is $b_{j}$. We also have $\theta_{j} \leq \theta_{j}^{\max }$. If $\theta_{j}^{*}=\left(R_{j}^{*}-b_{j}\right) / 2 a_{j} \leq \theta_{j}^{\max }$, we could obtain $R_{j}^{*} \leq 2 a_{j} \theta_{j}^{\max }+b_{j}$. If $\theta_{j}^{*}=\left(R_{j}-b_{j}\right) / 2 a_{j} \geq$ $\theta_{j}^{\max }$, thus $R_{j} \geq 2 a_{j} \theta_{j}^{\max }+b_{j}$ and $\theta_{j}^{*}=\theta_{j}^{\max }$. By substituting $\theta_{j}^{*}$ into the upper game. $U^{M E C}$ could be considered as a decreasing function about $R_{j}$. Thus, we have $R_{j}=2 a_{j} \theta_{j}^{\max }+b_{j}$. In general, $\theta_{j}^{*} \leq \theta_{j}^{\max }$ and $R_{j} \leq 2 a_{j} \theta_{j}^{\max }+b_{j}$. The upper bound of $R_{j}^{*}$ is $2 a_{j} \theta_{j}^{\max }+b_{j}$. The proof is now completed. The optimal utility of WPT nodes could also be obtained as:

$$
\begin{gathered}
U_{j}^{W P T *}=\frac{\left(R_{j}-b_{j}\right)^{2}}{4 a_{j}}-e_{j}, \forall j \in \mathcal{N} \\
\text { s.t. } \quad b_{j} \leq R_{j} \leq 2 a_{j} \theta_{j}^{\max }+b_{j}
\end{gathered}
$$

\section{B. Upper-Game of the MEC Node}

Definition 2 (SE): In upper-game, the economic incentive strategies $\boldsymbol{R}^{*}=\left(R_{1}^{*}, R_{2}^{*}, \ldots, R_{j}^{*}, \ldots, R_{N}^{*}\right)$ of the MEC node is the Stackelberg equilibrium (SE), if and only if $U_{j}^{W P T}\left(R_{j}^{*}, \theta_{j}, \Theta_{-j}\right) \leq U_{j}^{W P T}\left(R_{j}^{*}, \theta_{j}^{*}, \Theta_{-j}\right)$ and $U^{M E C}\left(\boldsymbol{R}, \boldsymbol{\theta}^{*}\right) \leq U^{M E C}\left(\boldsymbol{R}^{*}, \boldsymbol{\theta}^{*}\right)$.

Theorem 2: There exists a unique Stackelberg equilibrium between the leader and followers in our proposed game.

Proof: We will first substitute Eq. (16) into P2. P2 in the upper game could be rewritten as follows:

$$
\begin{aligned}
\max _{(\boldsymbol{R})} U^{M E C}= & \sum \xi_{i} \ln \left[T_{i}^{\max }-\frac{\rho_{i}}{B \log _{2}\left(1+\frac{\kappa_{i} H_{i}}{2 a_{j} N_{0} B} \sum h_{j i}\left(R_{j}-b_{j}\right)\right)}\right. \\
& \left.-\mathcal{O}\left(\log \left(\frac{1}{\delta_{i}}\right)\right) \sqrt[3]{\frac{2 a_{j} \mu_{i} c_{i}^{3} d_{i}^{3}}{\kappa_{i} \sum h_{j i}\left(R_{j}-b_{j}\right)}}+1\right] \\
& -\sum R_{j}\left(R_{j}-b_{j}\right) / 2 a_{j} \\
\text { s.t. } & b_{j} \leq R_{j} \leq b_{j}+2 a_{j} \theta_{j}^{\max }, \forall j \in \mathcal{N} \\
& \theta_{j}^{\min } \leq \theta_{j} \leq \theta_{j}^{\max }, \forall j \in \mathcal{N} \\
& 0<\delta_{i}<1, \forall i \in \mathcal{M}
\end{aligned}
$$

In the above P2, we could easily observe that each term in the second summation is a quadratic function with respect to $\boldsymbol{R}$ (i.e., convex function). So we only need to investigate the first summation of the utility function $U^{M E C}$. In the first summation, each term represents the satisfaction degree $S_{i}(\boldsymbol{R})=\xi_{i} \ln \left(1+\varphi_{i}(\boldsymbol{R})\right)$. We now find the first derivative and the second derivative of $S_{i}(\boldsymbol{R})$ with regard to $\boldsymbol{R}$ :

$$
\begin{gathered}
\frac{\partial S_{i}}{\partial R_{m}}=\xi_{i} \frac{1}{1+\varphi_{i}(\boldsymbol{R})} \frac{\partial \varphi_{i}(\boldsymbol{R})}{\partial R_{m}} \\
\frac{\partial^{2} S_{i}}{\partial^{2} R_{m}}=\frac{-\xi_{i}}{\left(1+\varphi_{i}\right)^{2}} \frac{\partial \varphi_{i}}{\partial R_{m}} \frac{\partial \varphi_{i}}{\partial R_{m}}+\frac{\xi_{i}}{1+\varphi_{i}} \frac{\partial \varphi_{i}^{2}}{\partial^{2} R_{m}} \\
\frac{\partial^{2} S_{i}}{\partial R_{m} \partial R_{n}}=\frac{-\xi_{i}}{\left(1+\varphi_{i}\right)^{2}} \frac{\partial \varphi_{i}}{\partial R_{m}} \frac{\partial \varphi_{i}}{\partial R_{n}}+\frac{\xi_{i}}{1+\varphi_{i}} \frac{\partial \varphi_{i}^{2}}{\partial R_{m} \partial R_{n}}
\end{gathered}
$$




$$
\begin{gathered}
H_{i}=-\left[\frac{\xi_{i}}{\left(1+\varphi_{i}(\boldsymbol{R})\right)^{2}} X_{i}^{2}+\frac{\xi_{i}}{1+\varphi_{i}(\boldsymbol{R})} Y_{i}\right]\left(\begin{array}{cccc}
h_{1 i} h_{1 i} & h_{1 i} h_{2 i} & \cdots & h_{1 i} h_{N i} \\
h_{2 i} h_{1 i} & h_{2 i} h_{2 i} & \cdots & h_{2 i} h_{N i} \\
\vdots & \vdots & \ddots & \vdots \\
h_{N i} h_{1 i} & h_{N i} h_{2 i} & \cdots & h_{N i} h_{N i}
\end{array}\right)_{N \times N} \quad, \quad \forall i \in \mathcal{M} \\
\frac{\partial \varphi_{i}(\boldsymbol{R})}{\partial R_{m}}=h_{m i}\left[\sqrt[3]{\frac{2\left(\mathcal{O}\left(\log \left(1 / \delta_{i}\right)\right)^{3} a_{j} \mu_{i} c_{i}^{3} d_{i}^{3}\right.}{27 \kappa_{i}\left(\sum h_{j i}\left(R_{j}-b_{j}\right)\right)^{4}}}+\frac{\rho_{i} \kappa_{i} H_{i} \ln 2}{2 a_{j} N_{0} B^{2}\left(1+S N R_{i}\right) \ln ^{2}\left(1+S N R_{i}\right)}\right]=h_{m i} X_{i} \\
\frac{\partial^{2} \varphi_{i}(\boldsymbol{R})}{\partial R_{m} \partial R_{m}}=-h_{m i} h_{m i}\left[\frac{4}{9} \sqrt[3]{\frac{2\left(\mathcal{O}\left(\log \left(1 / \delta_{i}\right)\right)^{3} a_{j} \mu_{i} c_{i}^{3} d_{i}^{3}\right.}{\kappa_{i}\left(\sum_{j} h_{j i}\left(R_{j}-b\right)\right)^{7}}}+\frac{\rho_{i} \kappa_{i}^{2} H_{i}^{2}\left(\ln \left(1+S N R_{i}\right)+2\right) \ln 2}{4 a_{j}^{2} N_{0}^{2} B^{3}\left(1+S N R_{i}\right)^{2} \ln ^{3}\left(1+S N R_{i}\right)}\right]=-h_{m i} h_{m i} Y_{i} \\
\frac{\partial^{2} \varphi_{i}(\boldsymbol{R})}{\partial R_{m} \partial R_{n}}=-h_{m i} h_{n i}\left[\frac{4}{9} \sqrt[3]{\frac{2\left(\mathcal{O}\left(\log \left(1 / \delta_{i}\right)\right)^{3} a_{j} \mu_{i} c_{i}^{3} d_{i}^{3}\right.}{\kappa_{i}\left(\sum_{j} h_{j i}\left(R_{j}-b\right)\right)^{7}}}+\frac{\rho_{i} \kappa_{i}^{2} H_{i}^{2}\left(\ln \left(1+S N R_{i}\right)+2\right) \ln 2}{4 a_{j}^{2} N_{0}^{2} B^{3}\left(1+S N R_{i}\right)^{2} \ln ^{3}\left(1+S N R_{i}\right)}\right]=-h_{m i} h_{n i} Y_{i}
\end{gathered}
$$

Where $R_{m}, R_{n} \in \boldsymbol{R}$ and $R_{m} \neq R_{n}$. In addition, we derive the first derivative and the second derivative of $\varphi_{i}(\boldsymbol{R})$ with regard to $\boldsymbol{R}$, which is shown at the top of next page. To simplify, we utilize $S N R_{i}=\frac{\kappa_{i} H_{i}}{2 a_{j} N_{0} B} \sum h_{j i}\left(R_{j}-b_{j}\right)$ to present the Signal-Noise Ratio during communication. By substituting Eq. (24)-(26) into Eq. (20)-(22), we have:

$$
\begin{gathered}
\frac{\partial^{2} S_{i}}{\partial^{2} R_{m}}=\frac{-\xi_{i}}{\left(1+\varphi_{i}\right)^{2}} h_{m i}^{2} X_{i}^{2}+\frac{-\xi_{i}}{1+\varphi_{i}} h_{m i}^{2} Y_{i} \\
\frac{\partial^{2} S_{i}}{\partial R_{m} \partial R_{n}}=\frac{-\xi_{i}}{\left(1+\varphi_{i}\right)^{2}} h_{m i} h_{n i} X_{i}^{2}+\frac{-\xi_{i}}{1+\varphi_{i}} h_{m i} h_{n i} Y_{i}
\end{gathered}
$$

Where $X_{i}>0$ and $Y_{i}>0, \forall i \in \mathcal{M}$. Thus, we could get the Hessian matrix $H_{i}$ of $S_{i}(\boldsymbol{R})$ through Eq. (27) (28), which is shown as Eq. (23). $-H_{i}$ is considered as a real symmetric matrix, which should be proved as a positive definite matrix, based on theorems in [43]. And we could prove $H_{i}$ is a strict negative definite matrix, which shows the existence and uniqueness of the optimal strategy $\boldsymbol{R}^{*}=$ $\left(R_{1}^{*}, R_{2}^{*}, \ldots, R_{j}^{*}, \ldots, R_{N}^{*}\right)$. Moreover, it also denotes that $\mathbf{P 2}$ is a convex optimization problem. The proof is now completed.

To find the unique Nash equilibrium (NE) and Stackelberg equilibrium (SE) in our game, we design a low complexity gradient-based searching algorithm as Algorithm 1.

\section{Optimal Learning Parameter Design}

Benefit from the optimal transmission power $\boldsymbol{\theta}^{*}$ from WPT nodes, a stable and robust federated edge learning process can be guaranteed in our framework. But the global learning efficiency is still related to learning accuracy $\alpha$ (global) and $\delta_{i}$ (local). To maximize the learning efficiency of our framework, we now study how to design the optimal parameters. We assume that all edge devices have the same local accuracy $\delta_{i}=\delta, \forall i \in \mathcal{M}$. Thus global federated learning time $T^{\text {total }}$ in our framework could be expressed as:

$$
T^{\text {total }}=\frac{\mathcal{O}(\log (1 / \alpha))}{1-\delta} \cdot \max \left\{T_{i}^{\text {learn }}\left(\delta, \boldsymbol{\theta}^{*}\right)\right\}
$$

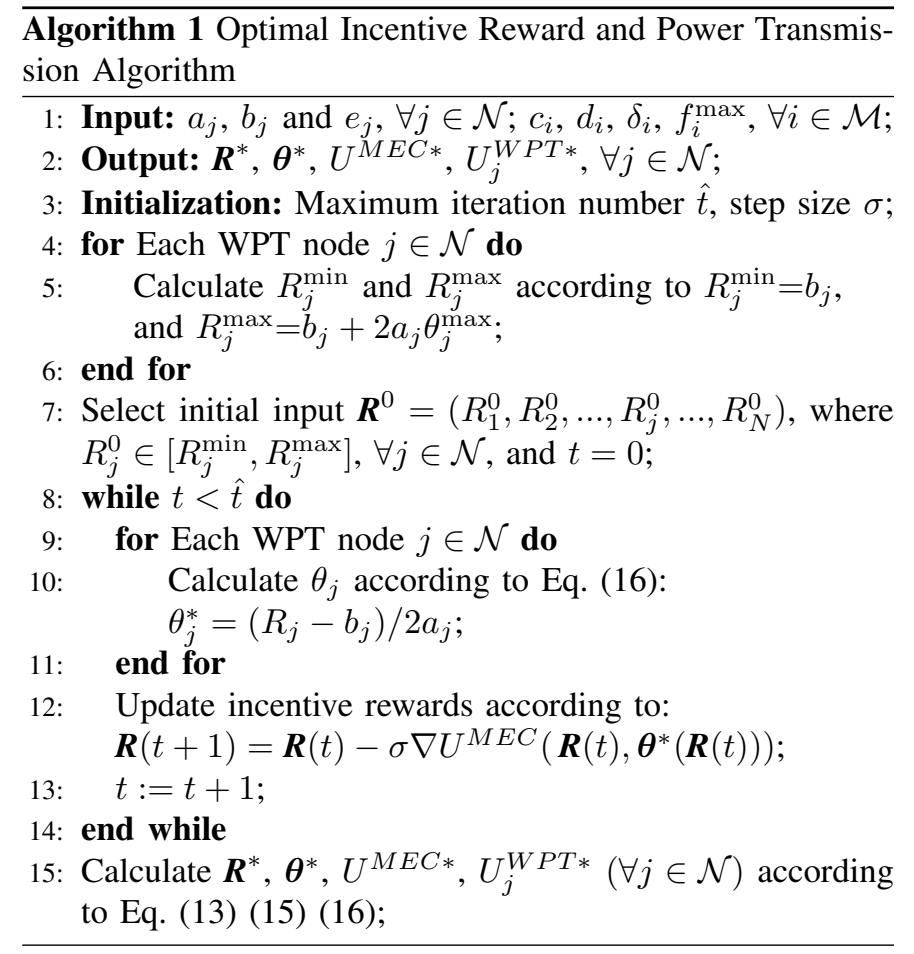

Let $t=\arg \max \left\{T_{i}^{\text {learn }}\left(\delta, \boldsymbol{\theta}^{*}\right)\right\}, t \in \mathcal{M}$ and $\mathcal{O}(\log (1 / \delta))=\beta \log (1 / \delta), \beta>0$. Thus we could formulate the optimization problem $\mathbf{P 3}$, which is shown as follows.

$$
\begin{gathered}
\min _{\delta} T^{\text {total }}=\frac{\mathcal{O}(\log (1 / \alpha))}{1-\delta}\left[T_{t}^{c o m}+\log \left(\frac{1}{\delta}\right) Z_{t}\left(\boldsymbol{\theta}^{*}\right)\right] \\
\text { s.t. } \quad 0<\alpha<1 ; 0<\delta<1
\end{gathered}
$$

Where $T_{t}^{c o m}\left(\boldsymbol{\theta}^{*}\right)$ is the communication time of the edge device $t$, and $Z_{t}\left(\boldsymbol{\theta}^{*}\right)=\beta \sqrt[3]{\frac{\mu_{t} c_{t}^{3} d_{t}^{3}}{\kappa_{t} \sum h_{j t} \theta_{j}^{*}}}, \forall j \in \mathcal{N}$.

Theorem 3: Under the optimal transmission power strategy $\boldsymbol{\theta}^{*}, \mathbf{P 3}$ is a the convex problem and there exists a unique local training accuracy $\delta^{*}$ in our framework.

Lemma 2: $\log (1 / \delta)>0.5(1-\delta)(3-\delta), \quad \delta \in(0,1)$ 
Proof: Let $G(\delta)=0.5(1-\delta)(3-\delta)-\log (1 / \delta)$, the first derivative of $G(\delta)$ with regard to $\delta$ is:

$$
\frac{\partial G(\delta)}{\partial \delta}=\delta+\frac{1}{\delta}-2>0, \quad \delta \in(0,1)
$$

Thus, $G(\delta)<G(1)=0, \delta \in(0,1)$. The proof is completed. Let $A=\mathcal{O}(\log (1 / \alpha))>0, \alpha \in(0,1)$, we now find the first and the second derivative of $T^{\text {total }}(\delta)$ as:

$$
\begin{gathered}
\frac{\partial T^{\text {total }}}{\partial \delta}=\frac{A\left[T_{t}^{c o m}+Z_{t} \log (1 / \delta)\right]}{(1-\delta)^{2}}-\frac{A Z_{t}}{\delta(1-\delta)} \\
\frac{\partial^{2} T^{\text {total }}}{\partial^{2} \delta}=\frac{A\left[T_{t}^{c o m}+Z_{t} \log (1 / \delta)\right]}{0.5(1-\delta)^{3}}-\frac{A Z_{t}(3 \delta-1)}{\delta^{2}(1-\delta)^{2}}
\end{gathered}
$$

According to Lemma 2, we could have:

$$
\begin{aligned}
\frac{\partial^{2} T^{\text {total }}}{\partial^{2} \delta} & >\frac{A\left[T_{t}^{\text {com }}+0.5 Z_{t}(1-\delta)(3-\delta)\right]}{0.5(1-\delta)^{3}}-\frac{A Z_{t}(3 \delta-1)}{\delta^{2}(1-\delta)^{2}} \\
& =\frac{2 A T_{t}^{c o m}}{(1-\delta)^{3}}+\frac{A \beta Z_{t}(1-\delta)}{\delta^{2}}>0, \delta \in(0,1)
\end{aligned}
$$

Therefore, $\mathbf{P 3}$ is a strict convex optimization problem. The

\begin{tabular}{|c|c|}
\hline System parameters & Value setting \\
\hline Number of edge devices $\mathcal{M}$ & $M=[5,100]$ \\
\hline Number of WPT nodes $\mathcal{N}$ & $N=[5,50]$ \\
\hline $\begin{array}{l}\text { The transmission bandwidth of all the } \\
\text { edge devices } B\end{array}$ & $B=1 \mathrm{MHz}$ \\
\hline The noise power spectrum density $N_{0}$ & $-174 \mathrm{dBm} / \mathrm{Hz}$ \\
\hline Channel power gain $h_{i j}$ and $H_{i}$ & $32.44+20 \lg (d)$ \\
\hline Data size of local weights/gradients $\rho_{i}$ & $\rho_{i}=1 \mathrm{MB}$ \\
\hline $\begin{array}{l}\text { The CPU cycles number for one sam- } \\
\text { ple data training } c_{i}\end{array}$ & $\begin{array}{l}{[10,30]} \\
\text { cycles/bit }\end{array}$ \\
\hline Data size of local personal dataset $d_{i}$ & {$[25,35] \mathrm{MB}$} \\
\hline $\begin{array}{l}\text { The local sub-model trained accuracy } \\
\delta_{i} \text { of edge devices }\end{array}$ & $\delta_{i} \in(0,1)$ \\
\hline $\begin{array}{l}\text { The transmission energy cost factors of } \\
\text { WPT nodes } a_{j} \text { and } b_{j}\end{array}$ & {$[0.6,0.2]$} \\
\hline $\begin{array}{l}\text { The maximum transmission power } \\
\theta_{j}^{\max } \text { of WPT nodes }\end{array}$ & $\theta_{j}^{\max }=[2,3] \mathrm{W}$ \\
\hline The energy harvesting efficiency $\kappa_{i}$ & $\kappa_{i}=[0.1,0.9]$ \\
\hline
\end{tabular}
proof is now completed. We could calculate the optimal $\delta^{*}$ via classic one-dimensional convex optimization search method with convex optimization tools (e.g., cvxopt).

TABLE II: System Parameters in WPEG Framework

\section{Simulation AND Discussion}

In this section, we conduct simulation experiments of our WPEG framework. Our experimental results mainly divide into three parts: 1) we compare our proposed optimal economic incentive strategy and power transmission strategy with several classic schemes in terms of the utility of the MEC node and the utility of WPT nodes, respectively; 2) we also study the influence of the number of edge devices, the number of WPT nodes, the energy harvesting efficiency on the performance of our framework; 3) we investigate the impact of learning parameter design on the performance of global learning efficiency in our framework. We conduct the federated learning simulation based on [5]. Some of the important simulation parameters settings are from [5], which are listed in Table II. Our simulation is conducted on Python 3.7 (numpy 1.18 and cvxopt 1.1) which based on the hardware of the 3.20 GHz I7 Intel processor and 16 GB RAM.

\section{A. Experiment Setup in Our WPEG}

1) Federated edge learning parameters: For each edge devices $i(i \in \mathcal{M})$, we set the training size $d_{i}$ of each device as a uniform distribution in $25-35 \mathrm{MB}$, CPU cycle number of one sample training $c_{i}$ is a uniformly distributed in $10-30$ cycles/bit, the maximum computing resources of each device $f_{i}^{\max }$ is uniformly distributed in $3.0-4.0 \mathrm{GHz}$. Furthermore, the data size of local weights and local gradients $\rho_{i}$ is $1 \mathrm{MB}$.

2) Wireless power transfer parameters: we simulate a $200 m \times 200 m$ square area with $M$ edge devices and $N$ WPT nodes. The number of edge devices $M$ is ranging from 5 to 100 and the number of WPT nodes $N$ is ranging from 5 to 50 . The MEC node is located at the center of the square area. Edge devices and WPT nodes are randomly distributed. Both of their horizontal and vertical coordinates follow the uniform distribution ranging from $-100 \mathrm{~m}$ to $100 \mathrm{~m}$. We also model the channel gain $h_{j i}$ and $H_{i}$ as the path loss model, i.e., $32.44+20 \log _{10}(d)$, where $d$ (meters) presents the distance in free space. The transmission bandwidth $B$ of all the edge devices is set as $1 \mathrm{MHz}$ and the noise power spectrum density $N_{0}$ is denoted as $-174 \mathrm{dBm} / \mathrm{Hz}$. The energy harvesting efficiency is set as $\kappa_{i}=[0.1,0.9]$, respectively. And the energy cost factors of WPT nodes are $a_{j}=0.6 \mathrm{~W}^{-2}$, and $b_{j}=0.2 \mathrm{~W}^{-1}$. The maximum transmission power $\theta_{j}^{\max }$ is a uniformly distributed in $2.0-3.0 \mathrm{~W}$.

\section{B. Comparison Analysis with Other Learning Strategies}

We compare our optimal learning strategy (i.e., the power transmission strategy and economic incentive strategy) with two different classic strategies, including random federated edge learning (RFEL) scheme and uniform federated edge learning (UFEL) scheme in terms of the utility of both parties in our framework.

- Random federated edge learning (UFEL) scheme: 1) The MEC node randomly provides economic incentives $R_{j}^{r}$ to WPT nodes from $\left[R_{j}^{\min }, R_{j}^{\max }\right](\forall j \in \mathcal{N})$ based on Eq. (19), and WPT nodes power edge devices with the optimal power transmission strategy $\theta_{j}^{*}$ according to Eq. (16); 2) The MEC node rewards WPT nodes with the optimal economic incentive strategy $R_{j}^{*}$, while WPT nodes randomly determine the power transmission strategy from $\left(0, \theta_{j}^{\max }\right](\forall j \in \mathcal{N})$.

- Uniform federated edge learning (UELS) scheme: 1) The MEC node uniformly select economic incentives $R_{j}^{u}$ to 


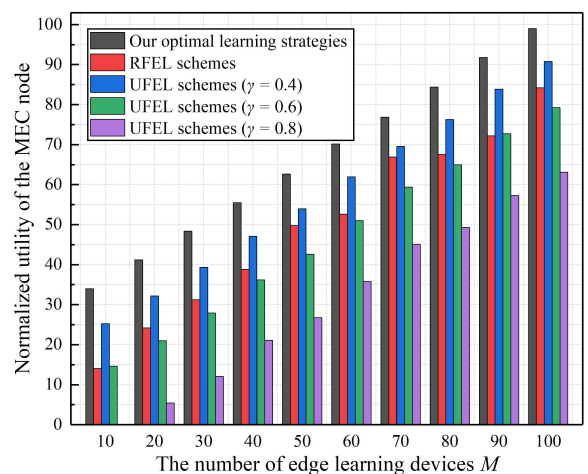

(a)

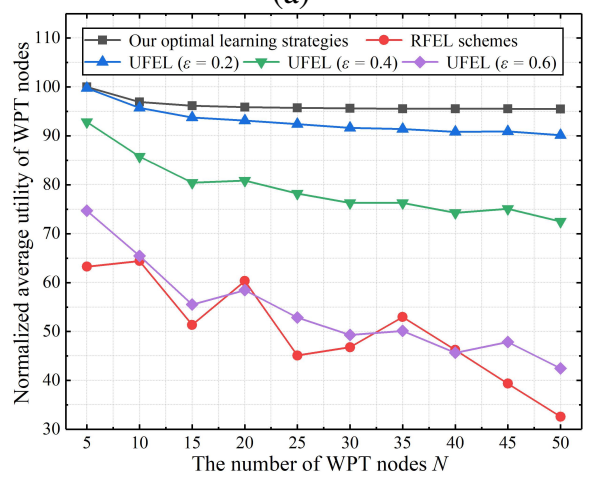

(d)

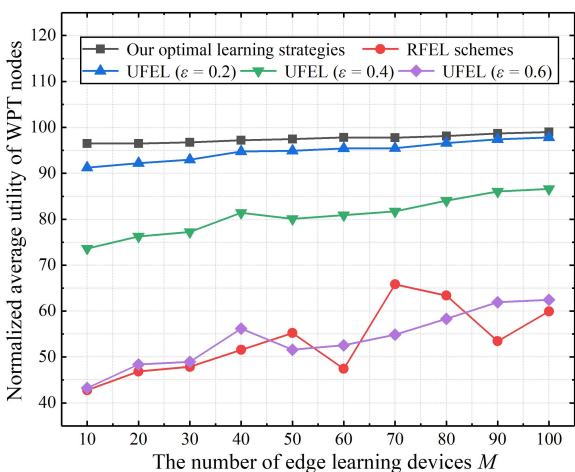

(b)

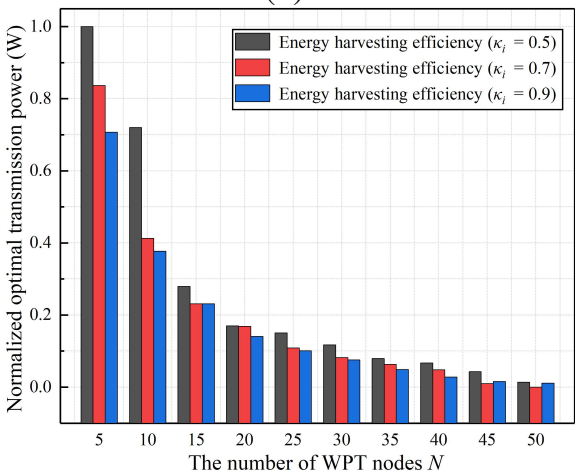

(e)

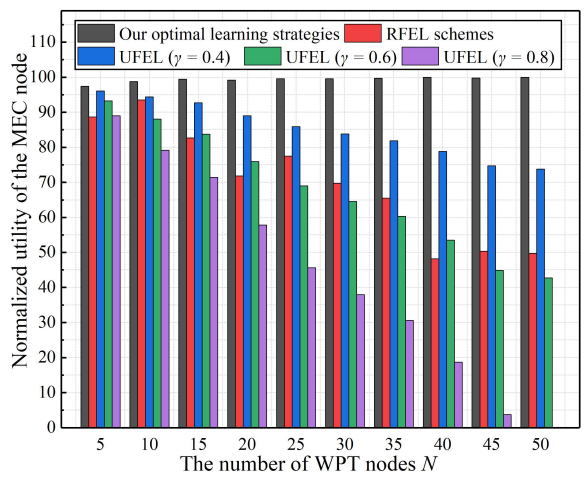

(c)

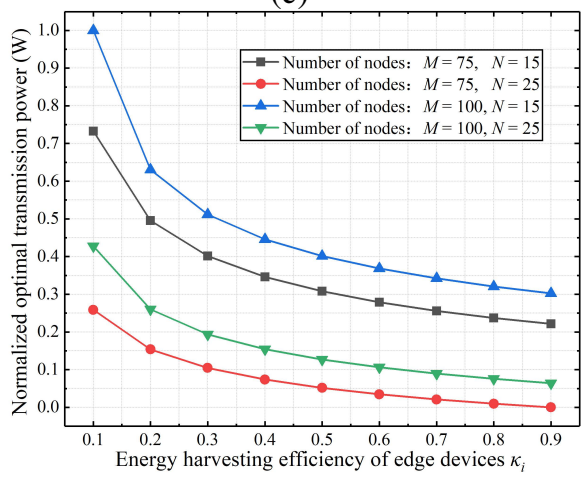

(f)

Fig. 4: (a) MEC node utility vs edge device number; (b) WPT node utility vs edge device number; (c) MEC node utility vs WPT node number; (d) WPT node utility vs WPT node number; (e) Transmission power vs WPT node number; (f) Transmission power vs energy harvesting efficiency

WPT nodes as $R_{j}^{u}=R_{j}^{\min }+\gamma\left(R_{j}^{\max }-R_{j}^{\min }\right)(\forall j \in \mathcal{N})$, where $\gamma=0.4,0.6,0.8$, respectively, and WPT nodes charge edge devices with the optimal power transmission strategy $\theta_{j}^{*}$;2) The MEC node grants WPT nodes with the optimal economic incentive strategy $R_{j}^{*}$, while WPT nodes uniformly adopt the power transmission strategy as $\theta_{j}^{u}=\theta_{j}^{\min }+\varepsilon\left(\theta_{j}^{\max }-\theta_{j}^{\min }\right)(\forall j \in \mathcal{N})$, where $\varepsilon=$ $0.2,0.4,0.6$, respectively.

We evaluate the performance of the above three learning strategies in terms of the utility of the MEC node and the average utility of WPT nodes, respectively, which are shown in Fig. 4. (a)-(d).

Specifically, Fig. 4. (a) (c) evaluate the economic pricing strategy. As shown in Fig. 4. (a), with the increase of edge learning devices, the utility of the MEC node has increased significantly. Because more devices bring more valuable data in federated learning, which improves the learning satisfaction of federated learning. Meanwhile, in terms of the average utility of the MEC node, compared with the RFEL and UFEL schemes, our optimal economic incentive strategy has increased by $32.38 \%, 14.42 \%, 41.37 \%$, and $110.19 \%$, respectively. Fig. 4. (c) evaluates the utility of MEC nodes as the number of WPT nodes changes. In our optimal strategy, the utility of the MEC node is relatively stable. This shows that simply increasing the number of WPT nodes could not improve the learning satisfaction of the MEC node. In this case, our optimal economic incentive strategy is improved by
$42.35 \%, 16.70 \%, 46.94 \%$, and $128.80 \%$ compared with the RFEL and UFEL schemes.

Fig. 4. (b) (d) investigate the power transmission strategy. As the number of edge devices increases, the utility of WPT nodes gradually increases as shown in Fig. 4. (b). This is because more edge devices require more energy to support the federated learning task. It will make MEC nodes trade more energy, which increases the utility of all WPT nodes. Besides, compared with the RFEL and UFEL schemes, our optimal power transmission strategy has increased $82.67 \%$, $2.86 \%, 20.77 \%$, and $81.28 \%$, respectively. While the average utility of WPT nodes decreases when WPT nodes become more as shown in Fig. 4, (d). This is because when edge devices are fixed, the total energy required for learning is relatively fixed. When the number of WPT nodes increases, the average energy trading amount is lower. Thus, the average utility of WPT nodes is reduced. Similarly, our optimal power transmission strategy also improved by $91.55 \%, 3.52 \%$, $21.43 \%$, and $77.42 \%$ compared with the other two schemes.

\section{Impact of Energy Harvesting Efficiency on Our Framework}

In Fig. 4. (e) (f), we study the impact of energy harvesting efficiency $\kappa_{i}$ on our learning system. As shown in Fig. 4. (e) (f), as the energy harvesting efficiency increases, the average power transmission of WPT nodes will decrease. Because when the energy conversion efficiency of edge devices is greater, WPT nodes only need to transmit less energy to 


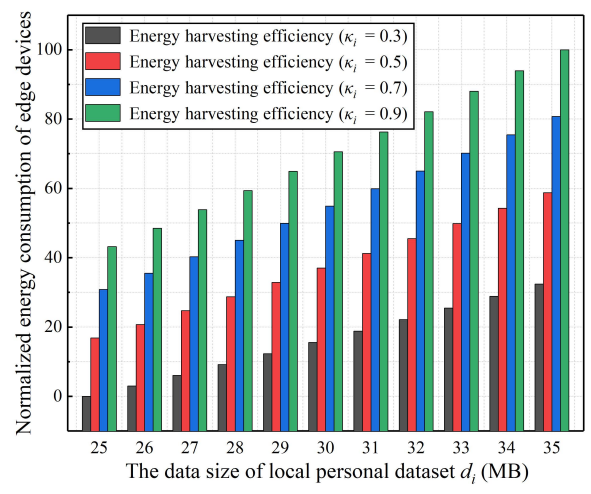

(a)

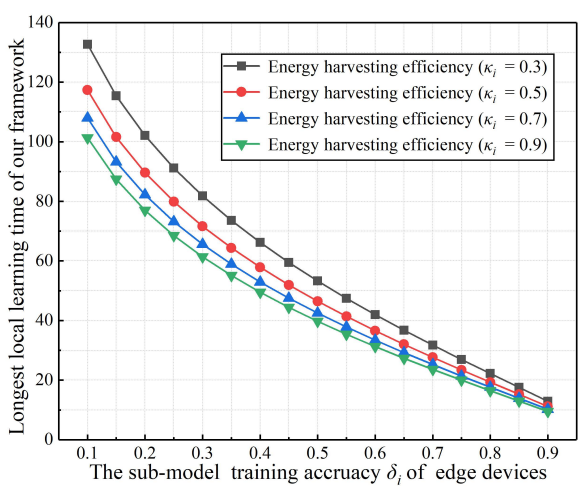

(b)

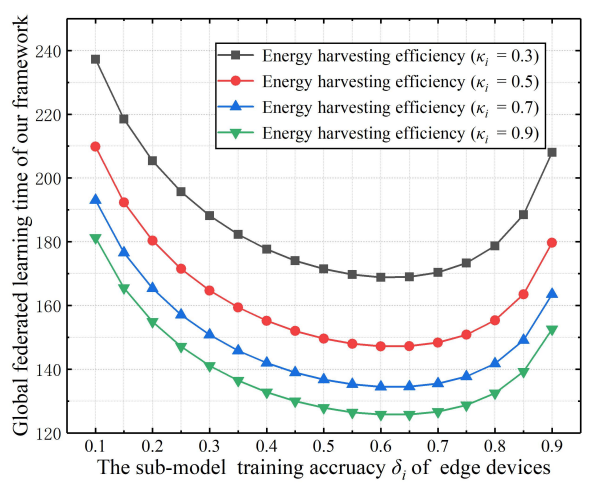

(c)

Fig. 5: (a) Energy consumption vs local dataset size; (b) local learning time vs local training accuracy; (c) Global learning time vs local training accuracy

meet the learning energy demands of edge devices. For WPT nodes, this also means the energy resource saving. At the same time, when the number of WPT nodes increases, the average transmission power of WPT nodes will further decrease. On the one hand, this is because the energy acquisition methods of edge devices are more decentralized and diversified, which reduces the transmission power of the single WPT node. On the other hand, in a limited spatial range, the denser WPT node deployment also means the average spatial distance between edge devices and WPT nodes is reduced. It will improve the energy channel gain and energy transmission efficiency, thus the transmission power of WPT nodes is reduced. When the number of edge devices is fixed and the number of WPT nodes is sufficient, the WPT nodes will charge edge devices according to the lowest power transmission strategy $\theta_{j}^{\min }$.

Meanwhile, as shown in Fig. 4. (f), when the number of edge devices decreases, the average power transmission of $\mathrm{W}$ PT nodes will also decrease accordingly. This is because when the number of edge devices is small, the energy requirement for learning is low. If the transmission power is too large and exceeds the energy demand of edge devices, edge devices can only adopt the largest computing resources $f_{i}^{\max }$ to learn, extra energy will be wasted. In this case, it is no longer energy resources that limits learning performance, but the computing resources of edge devices.

\section{Impact of Learning Parameters Design on Global Learning Performance}

In Fig. 5, we analyze the impact of learning parameter design on global learning performance in terms of learning energy consumption and learning time. As shown in Fig. 5. (a), as the local learning data size increases, the total learning energy consumption of edge devices will increase. Because when the local data size is larger, more local training is required, resulting in more energy consumption. However, the contribution of more data also means that edge devices will receive more rewards. We then show the relationship between local training accuracy and global learning time in Fig. 5. (b) (c). The global learning time, i.e., the total system efficiency, depends on global training accuracy $\alpha$, local training accuracy $\delta$, and the longest training time in each local iteration $T_{i}^{\max }$. We let $\mathcal{O}(\log (1 / \alpha))=\varpi \log (1 / \alpha)$, and make $\alpha=0.2$ and $\varpi=1$ without loss of generality. As shown in Fig. 5. (b), when the local accuracy $\delta$ increases, although the maximum time for local learning will be reduced, the corresponding local learning performance is reduced, resulting in an increase in the number of global iteration cycles. As shown in Fig. 5. (c), the local training accuracy and the global learning time are convexly related, that is, there is a unique trade-off to balance the local training accuracy and the global learning time. And we can easily obtain the optimal local accuracy $\delta^{*}$. In actual applications, we will adopt the optimal learning parameter design to maximize the efficiency of the system.

\section{CONCLUSION AND FutURE WORK}

In this paper, we have proposed a novel robust-efficient WPEG framework and investigated a joint optimization of power transfer, learning design, and user incentives for maximizing system efficiency and multi-user profits. In the framework, we have first employed a permissioned edge blockchain to manage the $\mathrm{P} 2 \mathrm{P}$ energy and knowledge sharing process in a secure and efficient manner. Besides, we have studied wirelessly-powered computing and communication models in edge AI, and characterized how wireless power transfer affects the latency and energy consumption of edge AI. Based on this, we have designed an energy-knowledge trading Stackelberg-game model to obtain the optimal power transmission and user incentives for our framework. Furthermore, the trade-off between the learning parameter and the global system efficiency has been studied and the uniqueness of the optimal learning parameter design has been proved. Numerical results have demonstrated that the game-based energy-knowledge incentive strategies could optimize the utilities of both parties in our framework, and attract WPT nodes charging edge devices to ensure robustefficient edge learning process. In addition, numerical results also have proved our optimal learning parameter design could achieve the optimal global learning efficiency. In future works, we will further consider user mobility with more effective incentives and resource allocation in our WPEG framework, which will enhance better network stability. 


\section{ACKNOWLEDGMENT}

This work is sponsored by the National Natural Science Foundation of China (Grant No. 61831007, 61972255).

\section{REFERENCES}

[1] C. Tseng et al., "Cloud-Based Artificial Intelligence System for LargeScale Arrhythmia Screening," in Computer, vol. 52, no. 11, pp. 40-51, Nov. 2019.

[2] S. Misra and G. Panwar, "5G Spectrum and Standards [Book Reviews]," in IEEE Wireless Communications, vol. 24, no. 1, pp. 4-5, February 2017.

[3] B. McMahan and D. Ramage. (Apr. 2017). Federated Learning: Collaborative Machine Learning Without Centralized Training Data. [Online]. Available: https://ai.googleblog.com/2017/04/federatedlearningcollaborative.html

[4] Z. Zhou, X. Chen, E. Li, L. Zeng, K. Luo and J. Zhang, "Edge Intelligence: Paving the Last Mile of Artificial Intelligence With Edge Computing," in Proceedings of the IEEE, vol. 107, no. 8, pp. 1738-1762, Aug. 2019.

[5] N. H. Tran, W. Bao, A. Zomaya, M. N. H. Nguyen and C. S. Hong, "Federated Learning over Wireless Networks: Optimization Model Design and Analysis," IEEE INFOCOM 2019 - IEEE Conference on Computer Communications, Paris, France, 2019, pp. 1387-1395.

[6] S. Wang et al., "Adaptive Federated Learning in Resource Constrained Edge Computing Systems," in IEEE Journal on Selected Areas in Communications, vol. 37, no. 6, pp. 1205-1221, June 2019.

[7] Y. Liu, J. J. Q. Yu, J. Kang, D. Niyato and S. Zhang, "Privacy-preserving Traffic Flow Prediction: A Federated Learning Approach," in IEEE Internet of Things Journal, doi: 10.1109/JIOT.2020.2991401.

[8] D. Ye, R. Yu, M. Pan and Z. Han, "Federated Learning in Vehicular Edge Computing: A Selective Model Aggregation Approach," in IEEE Access, vol. 8, pp. 23920-23935, 2020.

[9] Y. He, J. Ren, G. Yu and Y. Cai, "D2D Communications Meet Mobile Edge Computing for Enhanced Computation Capacity in Cellular Networks," in IEEE Transactions on Wireless Communications, vol. 18, no. 3, pp. 1750-1763, March 2019.

[10] K. Zhang, Y. Zhu, S. Leng, Y. He, S. Maharjan and Y. Zhang, "Deep Learning Empowered Task Offloading for Mobile Edge Computing in Urban Informatics," in IEEE Internet of Things Journal, vol.6, no.5, pp.7635-7647, Oct.2019.

[11] R. Zhang and C. K. Ho, "MIMO Broadcasting for Simultaneous Wireless Information and Power Transfer," in IEEE Transactions on Wireless Communications, vol. 12, no. 5, pp. 1989-2001, May 2013.

[12] H. Lee, K. Lee, H. Kim and I. Lee, "Wireless Information and Power Exchange for Energy-Constrained Device-to-Device Communications," in IEEE Internet of Things Journal, vol. 5, no. 4, pp. 3175-3185, Aug. 2018.

[13] Z. Zhou, M. Peng and Z. Zhao, "Joint Data-Energy Beamforming and Traffic Offloading in Cloud Radio Access Networks With Energy Harvesting-Aided D2D Communications," in IEEE Transactions on Wireless Communications, vol. 17, no. 12, pp. 8094-8107, Dec. 2018.

[14] M. M. Rana and W. Xiang, "IoT Communications Network for Wireless Power Transfer System State Estimation and Stabilization," in IEEE Internet of Things Journal, vol. 5, no. 5, pp. 4142-4150, Oct. 2018.

[15] S. Bi and Y. J. Zhang, "Computation Rate Maximization for Wireless Powered Mobile-Edge Computing With Binary Computation Offloading," in IEEE Transactions on Wireless Communications, vol. 17, no. 6, pp. 4177-4190, June 2018.

[16] L. Ji and S. Guo, "Energy-Efficient Cooperative Resource Allocation in Wireless Powered Mobile Edge Computing," in IEEE Internet of Things Journal, vol. 6, no. 3, pp. 4744-4754, June 2019.

[17] Y. Liu, K. Xiong, Q. Ni, P. Fan and K. B. Letaief, "UAV-Assisted Wireless Powered Cooperative Mobile Edge Computing: Joint Offloading, CPU Control, and Trajectory Optimization," in IEEE Internet of Things Journal, vol. 7, no. 4, pp. 2777-2790, April 2020.

[18] S. Gautam, T. X. Vu, S. Chatzinotas and B. Ottersten, "Cache-Aided Simultaneous Wireless Information and Power Transfer (SWIPT) With Relay Selection," in IEEE Journal on Selected Areas in Communications, vol. 37, no. 1, pp. 187-201, Jan. 2019.

[19] H. Zhang, Y. Wang, H. Ji and X. Li, "A Sleeping Mechanism for CacheEnabled Small Cell Networks With Energy Harvesting Function," in IEEE Transactions on Green Communications and Networking, vol. 4, no. 2, pp. 497-505, June 2020.
[20] S. Liao et al., "Cognitive Popularity based AI Service Sharing for Software-Defined Information-Centric Networks," in IEEE Transactions on Network Science and Engineering, doi: 10.1109/TNSE.2020.2993457.

[21] K. Zhang, S. Leng, X. Peng, P. Li, S. Maharjan, and Y. Zhang, "Artificial Intelligence Inspired Transmission Scheduling in Cognitive Vehicular Communications and Networks," in IEEE Internet of Things Journal, vol.6, no.2, pp.1987-1997, April 2019.

[22] E. Li, L. Zeng, Z. Zhou and X. Chen, "Edge AI: On-Demand Accelerating Deep Neural Network Inference via Edge Computing," in IEEE Transactions on Wireless Communications, vol. 19, no. 1, pp. 447-457, Jan. 2020.

[23] E. Li, Z. Zhou, and X. Chen, "Edge intelligence: On-demand deep learning model co-inference with device-edge synergy, in Proc. Workshop Mobile Edge Commun. (MECOMM SIGCOMM), Budapest, Hungary, Aug. 2018, pp. 3136.

[24] W. Liu, L. Chen, Y. Chen and W. Zhang, "Accelerating Federated Learning via Momentum Gradient Descent," in IEEE Transactions on Parallel and Distributed Systems, vol. 31, no. 8, pp. 1754-1766, 1 Aug. 2020.

[25] F. Sattler, S. Wiedemann, K. Mller and W. Samek, "Robust and Communication-Efficient Federated Learning From Non-i.i.d. Data," in IEEE Transactions on Neural Networks and Learning Systems, doi: 10.1109/TNNLS.2019.2944481

[26] Z. Zhou, S. Yang, L. J. Pu and S. Yu, "CEFL: Online Admission Control, Data Scheduling and Accuracy Tuning for Cost-Efficient Federated Learning Across Edge Nodes," in IEEE Internet of Things Journal, doi: 10.1109/JIOT.2020.2984332.

[27] X. Lin, J. Li, J. Wu, H. Liang and W. Yang, "Making Knowledge Tradable in Edge-AI Enabled IoT: A Consortium Blockchain-Based Efficient and Incentive Approach," in IEEE Transactions on Industrial Informatics, vol. 15, no. 12, pp. 6367-6378, Dec. 2019.

[28] Y. Lu, X. Huang, Y. Dai, S. Maharjan and Y. Zhang, "Blockchain and Federated Learning for Privacy-Preserved Data Sharing in Industrial IoT," in IEEE Transactions on Industrial Informatics, vol. 16, no. 6, pp. 4177-4186, June 2020.

[29] K. Zhang, Y. Zhu, S. Maharjan, and Y. Zhang, "Edge Intelligence and Blockchain Empowered 5G Beyond for Industrial Internet of Things", IEEE Network Magazine, vol.33, no.5, pp.12-19, Sep/Oct.2019.

[30] Y. Lu, X. Huang, Y. Dai, S. Maharjan and Y. Zhang, "Differentially Private Asynchronous Federated Learning for Mobile Edge Computing in Urban Informatics," in IEEE Transactions on Industrial Informatics, vol. 16, no. 3, pp. 2134-2143, March 2020.

[31] Y. Sarikaya and O. Ercetin, "Motivating Workers in Federated Learning: A Stackelberg Game Perspective," in IEEE Networking Letters, vol. 2, no. 1, pp. 23-27, March 2020.

[32] J. Kang, Z. Xiong, D. Niyato, S. Xie and J. Zhang, "Incentive Mechanism for Reliable Federated Learning: A Joint Optimization Approach to Combining Reputation and Contract Theory," in IEEE Internet of Things Journal, vol. 6, no. 6, pp. 10700-10714, Dec. 2019.

[33] Z. Yang, K. Yang, L. Lei, K. Zheng and V. C. M. Leung, "BlockchainBased Decentralized Trust Management in Vehicular Networks," in IEEE Internet of Things Journal, vol. 6, no. 2, pp. 1495-1505, April 2019.

[34] X. Lin, J. Wu, S. Mumtaz, S. Garg, J. Li and M. Guizani, "Blockchainbased On-Demand Computing Resource Trading in IoV-Assisted Smart City," in IEEE Transactions on Emerging Topics in Computing, doi: 10.1109/TETC.2020.2971831.

[35] Z. Li, J. Kang, R. Yu, D. Ye, Q. Deng and Y. Zhang, "Consortium Blockchain for Secure Energy Trading in Industrial Internet of Things," in IEEE Transactions on Industrial Informatics, vol. 14, no. 8, pp. 36903700, Aug. 2018.

[36] Q. Xu, Z. Su and Q. Yang, "Blockchain-Based Trustworthy Edge Caching Scheme for Mobile Cyber-Physical System," in IEEE Internet of Things Journal, vol. 7, no. 2, pp. 1098-1110, Feb. 2020.

[37] Z. Chang, W. Guo, X. Guo, Z. Zhou and T. Ristaniemi, "Incentive Mechanism for Edge-Computing-Based Blockchain," in IEEE Transactions on Industrial Informatics, vol. 16, no. 11, pp. 7105-7114, Nov. 2020.

[38] Z. Xiong, S. Feng, W. Wang, D. Niyato, P. Wang and Z. Han, "Cloud/Fog Computing Resource Management and Pricing for Blockchain Networks," in IEEE Internet of Things Journal, vol. 6, no. 3, pp. 4585-4600, June 2019.

[39] J. Chen et al., "Collaborative Trust Blockchain Based Unbiased Control Transfer Mechanism for Industrial Automation," in IEEE Transactions on Industry Applications, doi: 10.1109/TIA.2019.2959550.

[40] J. Koněcný, Z. Qu, and P. Richtárik, "Semi-stochastic coordinate descent," Optimization Methods and Software, vol. 32, no. 5, pp. 993-1005, Sep. 2017. 
[41] R. Du, H. Shokri-Ghadikolaei and C. Fischione, "Wirelessly-Powered Sensor Networks: Power Allocation for Channel Estimation and Energy Beamforming," in IEEE Transactions on Wireless Communications, vol. 19, no. 5, pp. 2987-3002, May 2020.

[42] L. Park, Y. Jang, S. Cho and J. Kim, "Residential Demand Response for Renewable Energy Resources in Smart Grid Systems," in IEEE Transactions on Industrial Informatics, vol. 13, no. 6, pp. 3165-3173, Dec. 2017.

[43] S. Boyd and L. Vandenberghe, Convex Optimization. Cambridge, U.K.: Cambridge Univ. Press, 2004

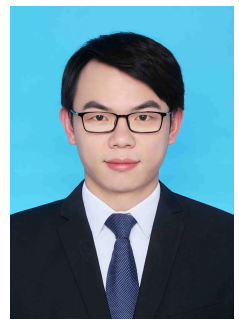

Xi Lin received the B.S. degree from the School of Precision Instrument and Opto-Electronics Engineering, Tianjin University, Tianjin, China, in 2016. $\mathrm{He}$ is currently pursuing the Ph.D. degree with the School of Electronic Information and Electrical Engineering, Shanghai Jiao Tong University, Shanghai, China. His research interests include multi-access edge computingInternet of Things, Blockchain, and so on. He is a Student Member of the IEEE.

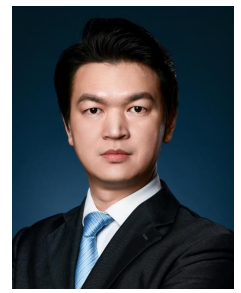

Jun Wu received the Ph.D. degree in information and telecommunication studies from Waseda University, Japan, in 2011. He was a Post-Doctoral Researcher with the Research Institute for Secure Systems, National Institute of Advanced Industrial Science and Technology (AIST), Japan, from 2011 to 2012. He was a Researcher with the Global Information and Telecommunication Institute, Waseda University, Japan, from 2011 to 2013 . He is currently an associate professor of School of Electronic Information and Electrical Engineering, Shanghai Jiao Tong University, China. He is also the vice director of National Engineering Laboratory for Information Content Analysis Technology, Shanghai Jiao Tong University, China. He is the chair of IEEE P21451-1-5 Standard Working Group. He has hosted and participated in a lot of research projects including National Natural Science Foundation of China (NFSC), National 863 Plan and 973 Plan of China, Japan Society of the Promotion of Science Projects (JSPS), etc. His research interests include the advanced computing, communications and security techniques of software-defined networks (SDN), informationcentric networks (ICN) smart grids, Internet of Things (IoT), 5G, etc., where he has published more than 120 refereed papers. He has been the Track Chair of VTC 2019 and the TPC Member of more than ten international conferences including ICC, GLOBECOM, WINCON, etc. He has been a Guest Editor of the IEEE Sensors Journal, Sensors, ICT Express. He is an Associate Editor of the IEEE Access.

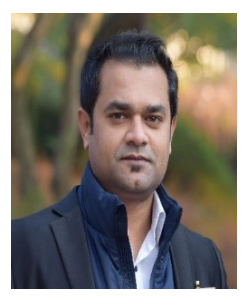

Ali Kashif Bashir is a Senior Lecturer at the Department of Computing and Mathematics, Manchester Metropolitan University, United Kingdom. He is also holding Adjunct Professor Position at National University of Science and Technology, Pakistan. He is a senior member of IEEE, invited member of IEEE Industrial Electronic Society, member of ACM, and Distinguished Speaker of ACM. His past assignments include Associate Professor of ICT, University of the Faroe Islands, Denmark; Osaka University, Japan; Nara National College of Technology, Japan; the National Fusion Research Institute, South Korea; Southern Power Company Ltd., South Korea, and the Seoul Metropolitan Government, South Korea. $\mathrm{He}$ has worked on several research and industrial projects of South Korean, Japanese and European agencies and Government Ministries. He received his Ph.D. in computer science and engineering from Korea University South Korea. He has authored over 120 research articles and is supervising/co-supervising several graduate (MS and $\mathrm{PhD}$ ) students. His research interests include internet of things, wireless networks, distributed systems, network/cyber security, cloud/network function virtualization, machine learning, etc. He is serving as the Editor-in-chief of the IEEE FUTURE DIRECTIONS NEWSLETTER.

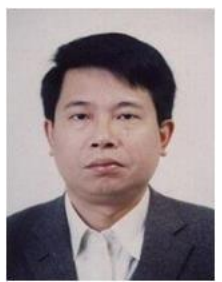

Jianhua $\mathbf{L i}$ is a professor and the dean of School of Cyber Security, Shanghai Jiao Tong University, Shanghai, China. He is also the director of National Engineering Laboratory for Information Content Analysis Technology, the director of Engineering Research Center for Network Information Security Management and Service of Chinese Ministry of Education, and the director of Shanghai Key Laboratory of Integrated Administration Technologies for Information Security, China. He is the vice president of Association of Cyber Security Association of China. He got his BS, MS and Ph.D. degrees from Shanghai Jiao Tong University, in 1986, 1991 and 1998, respectively. He was the chief expert in the information security committee experts of National High Technology Research and Development Program of China (863 Program) of China. He was the leader of more than 30 state/province projects of China, and published more than 300 papers. He published 6 books and has about 20 patents. He made 3 standards and has 5 software copyrights. He got the Second Prize of National Technology Progress Award of China in 2005. His research interests include information security, signal process, computer network communication, etc.

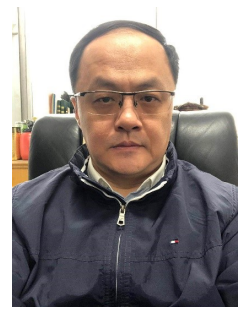

Wu Yang received the Ph.D. degree in computer system architecture from the Computer Science and Technology School, Harbin Institute of Technology. $\mathrm{He}$ is currently a Professor and a Doctoral Supervisor with Harbin Engineering University. His main research interests include wireless sensor networks, peer-to-peer networks, and information security. $\mathrm{He}$ is a member of ACM and a Senior Member of CCF.

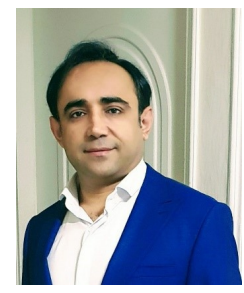

Md. Jalil Piran received the Ph.D. degree in electronics and radio engineering from Kyung Hee University, South Korea, in 2016. He was a PostDoctoral Research Fellow in resource management and quality of experience in $5 \mathrm{G}$ cellular networks and the Internet of Things (IoT) with the Networking Laboratory, KyungHee University. Dr. Jalil Piran is currently an Assistant Professor with the Department of Computer Science and Engineering, Sejong University, Seoul, South Korea. Prof. Piran has published a substantial number of technical papers in well-known international journals and conferences in research fields of: Wireless Communications; 5G and beyond, Internet of Things (IoT) Multimedia communication; streaming, adaptation, and QoE, Applied Machine learning, Security. 